\title{
Exact results for an STU-model
}

\author{
G.L. Cardoso, ${ }^{a}$ B. de $\mathbf{W i t}^{b, c}$ and S. Mahapatra ${ }^{d}$ \\ ${ }^{a}$ Center for Mathematical Analysis, Geometry and Dynamical Systems, \\ Department of Mathematics, Instituto Superior Técnico, Universidade de Lisboa, \\ Av. Rovisco Pais, Lisboa 1049-001, Portugal \\ ${ }^{b}$ Nikhef, Science Park 105, Amsterdam 1098 XG, The Netherlands \\ ${ }^{c}$ Institute for Theoretical Physics, Utrecht University, \\ Princetonplein 5, Utrecht 3584 CC, The Netherlands \\ ${ }^{d}$ Physics Department, Utkal University, \\ Bhubaneswar 751004 , India \\ E-mail: gcardoso@math.tecnico.ulisboa.pt, B.deWit@uu.nl, \\ swapna@iopb.res.in
}

ABSTRACT: The duality symmetries of the STU-model of Sen and Vafa are very restrictive. This is utilized to determine the holomorphic function that encodes its two-derivative Wilsonian effective action and its couplings to the square of the Weyl tensor to fifth order in perturbation theory. At fifth order some ambiguities remain which are expected to resolve themselves when proceeding to the next order. Subsequently, a corresponding topological string partition function is studied in an expansion in terms of independent invariants of $S, T$ and $U$, with coefficient functions that depend on an effective duality invariant coupling constant $u$, which is defined on a Riemann surface $\mathbb{C}$. The coefficient function of the invariant that is independent of $S, T$ and $U$ is determined to all orders by resummation. The other functions can be solved as well, either algebraically or by solving differential equations whose solutions have ambiguities associated with integration constants. This determination of the topological string partition function, while interesting in its own right, reveals new qualitative features in the result for the Wilsonian action, which would be difficult to appreciate otherwise. It is demonstrated how eventually the various ambiguities are eliminated by comparing the results for the effective action and the topological string. While we only demonstrate this for the leading terms, we conjecture that this will hold in general for this model.

KEYWORDS: Extended Supersymmetry, Supersymmetric Effective Theories, Supersymmetry and Duality, Topological Strings

ArXiv EPrint: 1907.04077 


\section{Contents}

1 Introduction 1

2 The STU-model and its dualities $\quad 4$

3 Higher-order contributions to the Wilsonian action 11

$\begin{array}{lll}4 & \text { The topological string partition function } & 17\end{array}$

5 Partial determination of the function $h \quad 22$

$\begin{array}{lll}6 & \text { Evaluating contributions contained in } h_{1} & 27\end{array}$

$\begin{array}{lll}7 & \text { Summary and conclusions } & 35\end{array}$

$\begin{array}{ll}\text { A The explicit expressions for } N_{I J} \text { and } N^{I J} & 37\end{array}$

B The structure of $\Omega$ in the large- $T$ and large- $U$ limit $\quad 37$

\section{Introduction}

For a general $N=2$ string compactification it is difficult to obtain exact expressions for the part of the Wilsonian effective action that describes the gravitational interactions with the vector multiplets, as well as for the topological string partition function. Often one has to make use of partial results obtained by concentrating on the neighbourhood of special points in the string moduli space, or from integrating the holomorphic anomaly equation for low genus. In this paper we want to investigate whether there exists a model for which the Wilsonian action and the topological string partition function can in principle be derived from their duality symmetries. The model we have in mind was discovered by Sen and Vafa when constructing dual pairs of type-II string compactifications in four space-time dimensions with $N=2$ supersymmetry [1]. These pairs were obtained by appropriate $\mathbb{Z}_{2} \times \mathbb{Z}_{2}$ orbifold constructions based on toroidally compactified type-II string theory. One such dual pair (referred to as $\mathrm{N}=2$ Example D) is described by an $N=2$ supergravity model with three vector multiplets and four hypermultiplets.

This model, which we will call the STU-model in the following, is the subject of study in this paper. It has a type-II description based on a Calabi-Yau three-fold with vanishing Euler number. In this description, the dilaton belongs to a hypermultiplet, and therefore the vector moduli space does not receive quantum corrections. The exact vector moduli space is based on an $[\mathrm{SL}(2) / \mathrm{SO}(2)]^{3}$ coset space with each factor modded out by the action of the integer-valued subgroup $\Gamma_{0}(2) \subset \mathrm{SL}(2 ; \mathbb{Z})$, defined by restricting its integer-valued 
matrix elements $a, b, c, d$ with $a d-b c=1$, by $a, d \in 2 \mathbb{Z}+1, c \in 2 \mathbb{Z}$ and $b \in \mathbb{Z}$. The quantum moduli space is therefore equal to $\left[\Gamma_{0}(2) \backslash \mathrm{SL}(2) / \mathrm{SO}(2)\right]^{3}$, and the vector multiplet sector is invariant under the product of an S-, a T- and a U-duality group, $\Gamma_{0}(2)_{\mathrm{S}} \times \Gamma_{0}(2)_{\mathrm{T}} \times \Gamma_{0}(2)_{\mathrm{U}}$. The role of the group $\Gamma_{0}(2)$ followed from the determination of the one-loop gravitational coupling that was computed explicitly by working at an orbifold point of the Calabi-Yau three-fold [2]. This coupling is invariant under $\left[\Gamma_{0}(2)\right]^{3}$ and involves the logarithm of a modular form that will play an important role in this paper. In addition, the model is also invariant under arbitrary permutations of the vector moduli $S, T$ and $U$. We will refer to this symmetry as triality. We stress that this is a different STU-model than the one studied, for instance, in [3], which is a reduction of the FHSV-model and whose duality group equals $\mathrm{SL}(2, \mathbb{Z})_{\mathrm{S}} \times \Gamma(2)_{\mathrm{T}} \times \Gamma(2)_{\mathrm{U}}$.

In this paper we will assume that the STU-duality symmetries (triality and $\left[\Gamma_{0}(2)\right]^{3}$ symmetry) remain valid at any order in perturbation theory, and we will use these symmetries to determine the holomorphic function [4] which encodes the part of the Wilsonian effective action that includes interactions proportional to the square of the Weyl tensor to fifth order in its gravitational coupling. Here it is important to stress that generically such functions do not transform as a function under duality transformations, and they are thus not straightforwardly invariant under the duality transformations of the moduli. Rather, the higher-order terms that one wishes to include should be such that the action of the electric-magnetic dualities on the field strengths and their duals will remain the same [5-8]. In the case at hand the holomorphic function will be expressed in terms of $\omega$, the logarithm of a (holomorphic) modular form that was identified in [2], and which depends on either one of the three complex moduli, $S, T$, or $U$, and its multiple holomorphic derivatives. At lowest non-trivial order duality invariance is achieved by allowing for the presence of a term $\ln X^{0}$ which, strictly speaking, is not part of the Wilsonian effective action. It is not unique, as there are non-holomorphic alternatives, and it has appeared at various stages in the literature. The fields $X^{0}, \mathrm{i} X^{0} S, \mathrm{i} X^{0} T$ and $\mathrm{i} X^{0} U$ are the complex scalar fields belonging to the four off-shell vector multiplets of the underlying supergravity.

From the holomorphic function and its complex conjugate one can derive a version of the topological string partition function of the STU-model by following the procedure outlined in [9]. ${ }^{1}$ This amounts to constructing the corresponding (real) Hesse potential by means of a suitable Legendre transformation. The Hesse potential depends on duality covariant moduli and it transforms as a function under general duality transformations, which for the STU-model constitute the group $\operatorname{Sp}(8 ; \mathbb{R})$. Consistency therefore requires that it is invariant under its $\left[\Gamma_{0}(2)\right]^{3}$ subgroup and under triality. The full Hesse potential is a real function, but as was demonstrated in [9], it decomposes into an infinite number of separately invariant functions of which precisely one exhibits the characteristic features of a topological string partition function. For instance, it is harmonic in the holomorphic modular form $\omega$, so that it will decompose into a sum of a function of $\omega$ and one of $\bar{\omega}$, but both these functions will still depend on the moduli and on their complex conjugates. The lack of homorphicity can be characterized in terms of a holomorphic anomaly equation,

\footnotetext{
${ }^{1}$ Actualy, the present study of this STU-model was partially motivated by the need to put the results of [9] to a test in the context of a realistic model.
} 
something that is a well-known feature of the topological string $[10,11]$. The holomorphic anomaly equation was generally derived in [9] on the basis of the diagrammatic structure in the Hesse potential. Upon including certain non-holomorphic terms for the genus-1 partition function, it was established that this anomaly equation belongs to the same class as the one that is known for the topological string. ${ }^{2}$ By explicit calculations one can then identify the leading terms with the low-genus topological string partition functions of the STU-model.

Subsequently we shall attempt to derive an all-order result for this topological string partition function. Here we follow an approach inspired by the work of [13], which enables us to resum a subclass of non-holomorphic terms by making use of the holomorphic anomaly equation. The result, which turns out to be qualitatively different from the result obtained in [13], can be further generalized by making it consistent with duality. This leads to a modified effective coupling constant $u$, which is duality invariant and depends on the moduli $S, T, U$. As it turns out, this effective coupling constant takes its values on a Riemann surface $\mathbb{C}$.

However, this result does not yet cover the full result for the topological string, because certain terms that have already been derived at low orders in perturbation theory, are not contained in the resummation. These terms are separately duality invariant and the obvious question is whether one can also extend them to all orders. As we will demonstrate, the coefficient functions, which depend on $u$, of terms that are products of three identical structures, whose arguments are equal to $S, T$, and $U$, respectively, satisfy differential equations that will lead to integration constants. Coefficient function of terms that are not of this type can be determined algebraically. However, the integration constants are directly related to specific terms in the holomorphic function that has been determined earlier. Given that the construction of this function can in principle be continued to all orders, it seems that the dualities of the STU-model indeed determine the holomorphic function and the corresponding topological string partition function.

This paper is organized as follows. In section 2 we introduce the main features of the STU-model and its dualities as well as the consequences of the STU-dualities for the holomorphic function, whose lowest-order contribution involves the logarithm of $X^{0}$. Various features of this logarithmic term as well as alternative versions are discussed. Section 3 describes the results for the holomorphic function up to fifth order in perturbation theory, which are obtained by imposing invariance under the dualities. We are not aware of any possible impediment for continuing this strategy to arbitrary orders of perturbation theory, and we will assume that this approach can in principle be continued to any given order.

Section 4 describes how to obtain the lowest-order results for a corresponding version of the topological string partition function, as well as its holomorphic anomaly equation, by following the method of [9]. This requires a Legendre transform, that was performed iteratively, leading to the so-called Hesse potential that is a function of duality covariant variables. One particular subsector takes the form of a topological string partition function

\footnotetext{
${ }^{2}$ It has been shown meanwhile that the holomorphic anomaly equation has an interpretation as an integrability condition for the existence of a Hessian structure [12].
} 
and transforms as a function under duality transformations. It depends holomorphically on the topological string coupling, with multiple covariant derivatives of the holomorphic modular forms $\omega(S), \omega(T)$, and $\omega(U)$. However, these covariant derivatives contain nonholomorphic connections, so that the result is not holomorphic in the moduli $S, T$, and $U$.

In section 5, we start the derivation of an all-order result for the topological string partition function. We first present a derivation of a function that captures all the nonholomorphic features of the topological string partition function, following an approach inspired by the work of [13]. After covariantizing this function one may collect the remaining terms into a second invariant function, whose leading terms in perturbation theory are now known and have a systematic structure in terms of a set of duality invariants.

Section 6 is then devoted to the determination of this last function, by imposing the holomorphic anomaly equation. Here we note that there are infinitely many different structures, as we are dealing with an infinite variety of invariants. Exploring the various terms we find that most of them can be determined algebraically in this way, while the remaining ones are subject to differential equations, which can be solved up to integration constants. Nevertheless it turns out that these integration constants can still be fixed by a careful comparison between the results for the topological string and those for the holomorphic function that encodes the Wilsonian effective action. This is one more indication that both the Wilsonian action and the topological string can be uniquely determined to all orders by requiring that the dualities act consistently.

The final section 7 presents our conclusions. We enclose a brief appendix A that contains a few useful formulae and a second appendix B in which we analyze the results in the limit where the real parts of two of the moduli are taken to infinity. In this case there are substantial simplifications.

\section{The STU-model and its dualities}

The effective action for the massless modes of the STU-model of Sen and Vafa can be described in terms of $N=2$ supergravity coupled to $n_{\mathrm{v}}=3$ vector multiplets and $n_{\mathrm{h}}=4$ hypermultiplets, so that the Euler characteristic $\chi \equiv 2\left(n_{\mathrm{v}}-n_{\mathrm{h}}+1\right)$ will vanish, as is required by the fact that the type-II description should be based on a self-mirror CalabiYau manifold. At the classical level its moduli space can be written as a product of the following special-Kähler and quaternion-Kähler spaces,

$$
\mathcal{M}_{\text {vector }}=\frac{\mathrm{SL}(2)}{\mathrm{SO}(2)} \times \frac{\mathrm{SL}(2)}{\mathrm{SO}(2)} \times \frac{\mathrm{SL}(2)}{\mathrm{SO}(2)}, \quad \mathcal{M}_{\text {hyper }}=\frac{\mathrm{SO}(4,4)}{\mathrm{SO}(4) \times \mathrm{SO}(4)} .
$$

In what follows we will focus on the vector multiplet sector.

The off-shell effective action for the massless fields is described in terms of four vector multiplets which contain four vector gauge fields, $W_{\mu}^{0}, W_{\mu}^{1}, W_{\mu}^{2}$ and $W_{\mu}^{3}$, as well as four complex scalars $X^{0}, X^{1}, X^{2}, X^{3}$. This description is locally superconformally invariant and therefore these fields are subject to local dilatations and phase transformations. As is well known, the Wilsonian effective action is encoded in a holomorphic function of these scalar fields that is homogeneous of second degree under complex scale transformations [4]. 
At the classical level this function takes the form

$$
F(X)=-\frac{X^{1} X^{2} X^{3}}{X^{0}},
$$

whose corresponding supergravity action leads precisely to the special-Kähler moduli space specified in (2.1). The isometry group of this moduli space is the direct product of three independent $\mathrm{SL}(2)$ groups, and not the product of their respective $\Gamma_{0}(2)$ subgroups. However, the function $F(X)$ will also contain terms that describe gravitational couplings of the special-Kähler moduli, which are only invariant under the direct product of the independent $\Gamma_{0}(2)$ groups [2]. Upon modding out the special-Kähler space in (2.1) by the action of the integer-valued group $\left[\Gamma_{0}(2)\right]^{3}$, the combined action will then be invariant under the latter group. Moreover, the invariance under permutations of the fields $X^{1}, X^{2}$ and $X^{3}$, known as triality, is respected by the function (2.2) as well as by its modification that we will introduce shortly.

The relevant special-Kähler moduli are conventionally denoted by $S, T$ and $U$, and defined by

$$
S=-\mathrm{i} \frac{X^{1}}{X^{0}}, \quad T=-\mathrm{i} \frac{X^{2}}{X^{0}}, \quad U=-\mathrm{i} \frac{X^{3}}{X^{0}} .
$$

These fields parametrize the special-Kähler target space and they are invariant under local dilatations and phase transformations. Since we intend to remain in the off-shell formulation we will retain the field $X^{0}$.

Since the supergravity description contains four vector gauge fields, one belonging to the Poincaré supergravity multiplet and one for each of the three matter multiplets, one will also be dealing with four magnetic charges denoted by $p^{0}, p^{1}, p^{2}, p^{3}$, and four electric charges denoted by $q_{0}, q_{1}, q_{2}, q_{3}$. These charges are carried by the underlying microscopic degrees of freedom of the STU-model. Under S-duality they transform as follows,

$$
\begin{array}{ll}
p^{0} \rightarrow d p^{0}+c p^{1}, & q_{0} \rightarrow a q_{0}-b q_{1}, \\
p^{1} \rightarrow a p^{1}+b p^{0}, & q_{1} \rightarrow d q_{1}-c q_{0}, \\
p^{2} \rightarrow d p^{2}-c q_{3}, & q_{2} \rightarrow a q_{2}-b p^{3}, \\
p^{3} \rightarrow d p^{3}-c q_{2}, & q_{3} \rightarrow a q_{3}-b p^{2} .
\end{array}
$$

In the STU-model the charges should take their values in an eight-dimensional discrete lattice that will only be invariant under the action of the $\Gamma_{0}(2)_{\mathrm{S}} \times \Gamma_{0}(2)_{\mathrm{T}} \times \Gamma_{0}(2)_{\mathrm{U}}$ duality group, so that the parameters $a, b, c$, and $d$ must be restricted accordingly. Based on the function (2.2), the moduli fields transform under $\Gamma_{0}(2)_{\mathrm{S}}$ as

$$
S \rightarrow \frac{a S-\mathrm{i} b}{d+\mathrm{i} c S}, \quad T \rightarrow T, \quad U \rightarrow U, \quad X^{0} \rightarrow(d+\mathrm{i} c S) X^{0} .
$$

Similar results apply to T- and U-duality transformations, which are directly obtained upon interchanging the labels $1 \leftrightarrow 2$ (or $1 \leftrightarrow 3$ ) and correspondingly $S \leftrightarrow T$ (or $S \leftrightarrow U$ ). From these transformation rules it follows that the eight charges will transform according to the $(\mathbf{2}, \mathbf{2}, \mathbf{2})$ representation of $\Gamma_{0}(2)_{\mathrm{S}} \times \Gamma_{0}(2)_{\mathrm{T}} \times \Gamma_{0}(2)_{\mathrm{U}}$.

As mentioned above, the function (2.2) will contain additional terms that break the original $[\mathrm{SL}(2)]^{3}$ dualities to the subgroup $\Gamma_{0}(2)_{\mathrm{S}} \times \Gamma_{0}(2)_{\mathrm{T}} \times \Gamma_{0}(2)_{\mathrm{U}}$. This must be done is 
such a way that the action of the duality subgroup on the field strengths and their duals will be preserved. Furthermore, as was already mentioned, the Wilsonian supersymmetric effective action for the STU-model must be encoded in a holomorphic function that is homogeneous of degree two [4], and should be consistent with the dualities as well as with triality. As it turns out, this puts stringent constraints on the way in which we can modify the function (2.2). To see this we start from the following holomorphic function,

$$
F(X, A)=-\frac{X^{1} X^{2} X^{3}}{X^{0}}+2 \mathrm{i} \Omega(X, A)
$$

where the first term describes the Lagrangian that is at most quadratic in space-time derivatives of the vector-multiplet fields. The extra term $\Omega$ contains a holomorphic parameter $A$, which actually corresponds to a field. This field $A$ is the lowest component of the square of a tensor chiral supermultiplet, known as the Weyl multiplet. Its presence in (2.6) will lead to higher-derivative interactions that involve among others the square of the Weyl tensor. Supersymmetry requires the function $F$ to be homogenous of degree two, i.e. $F\left(\lambda X, \lambda^{2} A\right)=\lambda^{2} F(X, A)$. The Wilsonian action will therefore be based on such a homogeneous holomorphic function. ${ }^{3}$

The duality transformations such as (2.4) are generated on the fields $X^{I}$ by electricmagnetic duality and this ensures that they will also act accordingly on the electric and magnetic charges. The Lagrangian and its underlying function $F(X, A)$ are therefore not invariant under the duality transformations. Rather the requirement is that the 'periods' $\left(X^{I}, F_{J}(X)\right)$ must transform covariantly under the dualities [4,5], precisely as the charges $\left(p^{I}, q_{J}\right)$ (see $\left.(2.4)\right)$. Hence the duality transformations involve the holomorphic derivatives of $F(X, A)$ which we list here for convenience (we refrain from indicating the dependence on $A$ for notational simplicity),

$$
\begin{aligned}
& F_{0}(X)=\frac{X^{1} X^{2} X^{3}}{\left(X^{0}\right)^{2}}-\frac{2 \mathrm{i}}{X^{0}}\left[-X^{0} \frac{\partial}{\partial X^{0}}+S \frac{\partial}{\partial S}+T \frac{\partial}{\partial T}+U \frac{\partial}{\partial U}\right] \Omega, \\
& F_{1}(X)=-\frac{X^{2} X^{3}}{X^{0}}+\frac{2}{X^{0}} \frac{\partial \Omega}{\partial S} \\
& F_{2}(X)=-\frac{X^{1} X^{3}}{X^{0}}+\frac{2}{X^{0}} \frac{\partial \Omega}{\partial T} \\
& F_{3}(X)=-\frac{X^{1} X^{2}}{X^{0}}+\frac{2}{X^{0}} \frac{\partial \Omega}{\partial U} .
\end{aligned}
$$

The above formulae clearly exhibit the triality symmetry, provided that $\Omega$ is triality invariant. The field $A$ is not subject to the duality transformations.

To construct the duality transformations on the fields, one considers the S-dualities acting on the charges $p^{I}$ and $q_{I}$ given in (2.4) and apply the same transformations on the

\footnotetext{
${ }^{3}$ We should point out that alternative higher-derivative couplings exist for these models, but they are not holomorphic $[14,15]$. Their behaviour under electric-magnetic duality has so far not been investigated in much detail and they do not contribute to the Wilsonian effective action nor to the topological string partition function.
} 
periods $X^{I}$ and $F_{I}$. The fields will thus transform as follows,

$$
\begin{aligned}
X^{0} \rightarrow X^{0 \prime} & =\Delta_{\mathrm{S}} X^{0}, & X^{1} \rightarrow X^{1 \prime} & =a X^{1}+b X^{0}, \\
X^{2} \rightarrow X^{2 \prime} & =\Delta_{\mathrm{S}} X^{2}-\frac{2 c}{X^{0}} \frac{\partial \Omega}{\partial U}, & X^{3} \rightarrow X^{3 \prime} & =\Delta_{\mathrm{S}} X^{3}-\frac{2 c}{X^{0}} \frac{\partial \Omega}{\partial T},
\end{aligned}
$$

where $a, b, c, d$ refer to the parameters of the S-duality transformation and $\Delta_{\mathrm{S}}(S)$ is defined by

$$
\Delta_{\mathrm{S}}(S)=d+\mathrm{i} c S
$$

Observe that there exist similar quantities $\Delta_{\mathrm{T}}(T)$ and $\Delta_{\mathrm{U}}(U)$ with parameters $d$ and $c$ that belong to the T- and U-duality transformations, respectively. Furthermore we note the convenient relations

$$
\frac{\partial S^{\prime}}{\partial S}=\frac{1}{\Delta_{S^{2}}}, \quad \frac{1}{S+\bar{S}} \rightarrow \frac{\left|\Delta_{\mathrm{S}}\right|^{2}}{S+\bar{S}}=\frac{\Delta_{\mathrm{S}}^{2}}{S+\bar{S}}-\Delta_{\mathrm{S}} \frac{\partial \Delta_{\mathrm{S}}}{\partial S} .
$$

The results lead to the following transformations of $X^{0}, S, T$ and $U$,

$$
\begin{aligned}
X^{0} \rightarrow X^{0 \prime} & =\Delta_{\mathrm{S}} X^{0}, \\
S \rightarrow S^{\prime} & =\frac{a S-\mathrm{i} b}{\Delta_{\mathrm{S}}}, \\
T & \rightarrow T^{\prime}=T+\frac{2}{\Delta_{\mathrm{S}}\left(X^{0}\right)^{2}} \frac{\partial \Delta_{\mathrm{S}}}{\partial S} \frac{\partial \Omega}{\partial U}, \\
U \rightarrow U^{\prime} & =U+\frac{2}{\Delta_{\mathrm{S}}\left(X^{0}\right)^{2}} \frac{\partial \Delta_{\mathrm{S}}}{\partial S} \frac{\partial \Omega}{\partial T},
\end{aligned}
$$

so that the S-duality transformations on $X^{0}$ and $S$ remain unchanged, while the fields $T$ and $U$ will now transform non-trivially. Obviously the T- and U-duality transformations follow from triality.

Because the STU-dualities are assumed to define symmetries of the model to all orders in perturbation theory, the transformation of the derivatives (2.7) must coincide with the results obtained by explicitly substituting the transformed values of the moduli given by (2.11) into the expressions for $F_{I}$,

$$
\begin{aligned}
& F_{0}\left(X^{\prime}\right)=a F_{0}(X)-b F_{1}(X), \\
& F_{1}\left(X^{\prime}\right)=d F_{1}(X)-c F_{0}(X), \\
& F_{2}\left(X^{\prime}\right)=a F_{2}(X)-b X^{3}, \\
& F_{3}\left(X^{\prime}\right)=a F_{3}(X)-b X^{2} .
\end{aligned}
$$

In that case the periods $\left(X^{I}, F_{J}(X)\right)$ transform covariantly under the dualities precisely as the charges $\left(p^{I}, q_{J}\right)$, as shown in (2.4). Note again that the function $F(X)$ itself does not transform as a function under electric-magnetic duality, which explains the precise form of the left-hand side of (2.12). The above equations (2.12) lead to conditions on the 
derivatives of $\Omega$ that take the following form,

$$
\begin{gathered}
\left(\frac{\partial \Omega}{\partial T}\right)_{\mathrm{S}}^{\prime}=\frac{\partial \Omega}{\partial T}, \quad\left(\frac{\partial \Omega}{\partial U}\right)_{\mathrm{S}}^{\prime}=\frac{\partial \Omega}{\partial U} \\
\left(\frac{\partial \Omega}{\partial S}\right)_{\mathrm{S}}^{\prime}-\Delta_{\mathrm{S}}^{2} \frac{\partial \Omega}{\partial S}=\frac{\partial \Delta_{\mathrm{S}}}{\partial S}\left[-\Delta_{\mathrm{S}} X^{0} \frac{\partial \Omega}{\partial X^{0}}-\frac{2}{\left(X^{0}\right)^{2}} \frac{\partial \Delta_{\mathrm{S}}}{\partial S} \frac{\partial \Omega}{\partial T} \frac{\partial \Omega}{\partial U}\right] \\
\left(X^{0} \frac{\partial \Omega}{\partial X^{0}}\right)_{\mathrm{S}}^{\prime} \\
=X^{0} \frac{\partial \Omega}{\partial X^{0}}+\frac{4}{\Delta_{\mathrm{S}}\left(X^{0}\right)^{2}} \frac{\partial \Delta_{\mathrm{S}}}{\partial S} \frac{\partial \Omega}{\partial T} \frac{\partial \Omega}{\partial U}
\end{gathered}
$$

These equations, which are non-linear in $\Omega$, were first derived in [6] for the more general case where $\Omega$ is not necessarily holomorphic. Note that the prime on the quantities on the left-hand side indicates that we have replaced all the fields by their transformed ones specified in (2.11). Corresponding results for T- and U-duality follow directly upon applying triality. As it turns out, the above equations are very restrictive, especially when insisting on triality. The function $\Omega$ can then be solved by iteration in powers of $A$, depending on some initial conditions. A noteworthy feature of the equations (2.13) is that they depend non-linearly on $\Omega$. The solution of these equations based on a power series in $A$ seems to be unique.

The iteration is based on the fact that $\Omega(X, A)$ must be a homogeneous function of second degree, which can be expanded in terms of a auxiliary complex field $A$ which scales with weight two. Because the fields $S, T, U$ do not scale, $X^{0}$ and $A$ are the only fields that are subject to scale transformations. Therefore $\Omega$ can be written in a series expansion in powers of $A\left(X^{0}\right)^{-2}$ with coefficient functions that depend on $S, T, U$ and an overall factor $A$,

$$
\Omega(X, A)=A\left[\gamma \ln \frac{\left(X^{0}\right)^{2}}{A}+\omega^{(1)}(S, T, U)+\sum_{n=1}^{\infty}\left(\frac{A}{\left(X^{0}\right)^{2}}\right)^{n} \omega^{(n+1)}(S, T, U)\right] .
$$

Note that we allowed for the presence of a logarithmic term, which under S-duality transforms with a shift proportional to $\ln \Delta_{\mathrm{S}}(S)$, with corresponding variations under $\mathrm{T}$ - and U-duality.

Let us now first concentrate on the lowest-order terms which should reproduce the result of [2]. Under S-duality one can directly analyze the equations (2.13) in lowest order in $A$. The last equation is trivially satisfied, whereas the other three equations give rise to the following restrictions on $\omega^{(1)}(S, T, U)$,

$$
\begin{aligned}
& \left(\frac{\partial \omega^{(1)}}{\partial T}\right)_{\mathrm{S}}^{\prime}=\frac{\partial \omega^{(1)}}{\partial T}, \quad\left(\frac{\partial \omega^{(1)}}{\partial U}\right)_{\mathrm{S}}^{\prime}=\frac{\partial \omega^{(1)}}{\partial U}, \\
& \left(\frac{\partial \omega^{(1)}}{\partial S}\right)_{\mathrm{S}}^{\prime}=\Delta_{\mathrm{S}}(S)^{2} \frac{\partial \omega^{(1)}}{\partial S}-2 \gamma \Delta_{\mathrm{S}} \frac{\partial \Delta_{\mathrm{S}}}{\partial S}
\end{aligned}
$$

Upon imposing triality the combined equations show that

$$
\omega^{(1)}(S, T, U)=\omega(S)+\omega(T)+\omega(U),
$$


where $\omega(S)$ must be the logarithm of a modular form which must transform as

$$
\omega\left(S^{\prime}\right)=\omega(S)-2 \gamma \ln \Delta_{\mathrm{S}}(S)
$$

Here we note that the transformation of the function $\omega(S)$ is in principle not fully captured by (2.17), because the right-hand side may also include a constant imaginary shift due to the multiplyer system of the modular form. Such a shift, if present, is harmless. The reason is that an imaginary constant shift leads to a real constant times the imaginary part of $A$ in the effective action. However, the imaginary part of $A$ encodes a Lagrangian that equals a total derivative, which can be ignored. Beyond the lowest order we will only be dealing with derivatives of $\omega(S)$, so that this imaginary shift is no longer relevant.

Obviously similar results hold for $\omega(T)$ and $\omega(U)$ by triality. Upon comparing (2.17) to the result found in [2] for the one-loop gravitational coupling in the STU-model, we must choose $\omega(S)$ equal to

$$
\omega(S)=\frac{1}{64 \pi} \ln \vartheta_{2}(S), \quad \text { with } \quad \gamma=-\frac{1}{256 \pi},
$$

whose corresponding duality group is precisely $\Gamma_{0}(2) .{ }^{4}$ We note that $\vartheta_{2}(S)$ can be expressed in terms of the Dedekind function as $\vartheta_{2}(S)=2 \eta^{2}(2 S) / \eta(S)$. Hence the choice (2.18) restricts the STU-dualities to the $\Gamma_{0}(2)$ subgroups of the generic SL(2) duality groups, so that the matrix elements $a, b, c, d$ should satisfy the restrictions pointed out in section 1 .

The fact that $\gamma$ is different from zero is important for the iteration that will be performed in the next section. In this iteration the quantity $A /\left(X^{0}\right)^{2}$ will play the role of a coupling 'constant', which is invariant under local dilatations and phase transformations, but not under the dualities. In the result of [2], the field $X^{0}$ is not present, so the shift in the variation of $\omega(S)$ has to be cancelled by some other term, such as

$$
\begin{aligned}
-2 \gamma \ln [(S+\bar{S})(T+\bar{T})(U+\bar{U})] \stackrel{\mathrm{S}}{\longrightarrow} & -2 \gamma \ln [(S+\bar{S})(T+\bar{T})(U+\bar{U})] \\
& +2 \gamma \ln \Delta_{\mathrm{S}}(S)+2 \gamma \ln \bar{\Delta}_{\mathrm{S}}(\bar{S}),
\end{aligned}
$$

and likewise for $\mathrm{T}$ - and U-duality. Obviously these variations are identical to those of $\gamma\left[\ln \left[\left(X^{0}\right)^{2} / A\right]+\right.$ h.c. $]$. However, within the context of the effective action the variation (2.19) is only an approximation, because the transformations of the moduli are corrected in view of (2.11). Furthermore it does not make sense to add a term to the effective action that explicitly involves the moduli, whose duality transformations are actually governed by the period vector $\left(X^{I}, F_{J}\right)$, where $F_{J}$ is defined as the derivative of the full function $F(X)$ with respect to $X^{J}$.

The above situation is, however, not problematic, because the big moduli space that involves the field $X^{0}$ is still subject to local dilatations as well as $\mathrm{U}(1)$ gauge transformations and there exists a gauge condition that replaces $\ln \left|X^{0}\right|$ in terms of a non-holomorphic term

\footnotetext{
${ }^{4}$ We note that while the expression for $\omega^{(1)}(S, T, U)$ given in (2.16) has manifest triality symmetry, the corresponding expression given in equation (2.6) of [2] involves both $\ln \vartheta_{2}$ and $\ln \vartheta_{4}$. However, by applying the modular transformation $\tau \rightarrow-1 / \tau$ to $\ln \vartheta_{4}$, the expression given in [2] becomes manifestly triality symmetric, and it agrees with the expression for $\omega^{(1)}(S, T, U)$ given in (2.16).
} 
whose duality transformation agrees in leading order with (2.19). To see this we introduce a generalized Kähler potential $\mathcal{K}$, defined by

$$
\mathcal{K}-\ln \left|X^{0}\right|^{2}=-\ln \left[\mathrm{i} \bar{X}^{I} F_{I}(X)-\mathrm{i} \bar{F}_{I}(\bar{X}) X^{I}\right],
$$

where the right-hand side is manifestly duality invariant and transforms under local dilatations. If we now impose a gauge condition for local dilatations by constraining $\left[\mathrm{i} \bar{X}^{I} F_{I}(X)-\right.$ $\left.\mathrm{i} \bar{F}_{I}(\bar{X}) X^{I}\right]$ to a real constant, then $\ln \left|X^{0}\right|$ is equal to a non-holomorphic expression whose leading contribution coincides with (2.19),

$$
\mathcal{K}=-\ln [(S+\bar{S})(T+\bar{T})(U+\bar{U})]+\mathcal{O}(\Omega) .
$$

Obviously it is convenient to work with the field $X^{0}$ throughout the calculations and to postpone imposing this gauge choice until the end. ${ }^{5}$

We should add that the logarithmic term $\ln X^{0}$ has appeared at various stages in the literature in the study of BPS black hole entropy. It was first discussed in [16] using the Gopakumar-Vafa term $[17,18]$ when calculating black hole entropy corrections. At the black hole horizon $A /\left(X^{0}\right)^{2}$ is inversely proportional to the square of the charges as a result of the BPS attractor equations [19]. The factor appears in the measure of an OSVtype integral derived in [20] for BPS black holes. The presence of this term is moreover consistent with the result of the logarithmic corrections to black hole entropy [21]. We refer to section 4.2.4 in [22] for further comments regarding this logarithmic term and its possible origin.

Before moving to the next section we discuss some technical aspects regarding the duality transformations of derivatives of $\omega(S)$ under S-duality. We list the first few multiple derivatives,

$$
\begin{aligned}
& \frac{\partial \omega}{\partial S} \rightarrow \Delta_{\mathrm{S}}{ }^{2} \frac{\partial \omega}{\partial S}-2 \gamma \Delta_{\mathrm{S}} \frac{\partial \Delta_{\mathrm{S}}}{\partial S} \\
& \frac{\partial^{2} \omega}{\partial S^{2}} \rightarrow \Delta_{\mathrm{S}}{ }^{4} \frac{\partial^{2} \omega}{\partial S^{2}}+2 \Delta_{\mathrm{S}}{ }^{3} \frac{\partial \Delta_{\mathrm{S}}}{\partial S} \frac{\partial \omega}{\partial S}-2 \gamma \Delta_{\mathrm{S}}{ }^{2}\left(\frac{\partial \Delta_{\mathrm{S}}}{\partial S}\right)^{2} \\
& \frac{\partial^{3} \omega}{\partial S^{3}} \rightarrow \Delta_{\mathrm{S}}{ }^{6} \frac{\partial^{3} \omega}{\partial S^{3}}+6 \Delta_{\mathrm{S}}{ }^{5} \frac{\partial \Delta_{\mathrm{S}}}{\partial S} \frac{\partial^{2} \omega}{\partial S^{2}}+6 \Delta_{\mathrm{S}}{ }^{4}\left(\frac{\partial \Delta_{\mathrm{S}}}{\partial S}\right)^{2} \frac{\partial \omega}{\partial S}-4 \gamma \Delta_{\mathrm{S}}{ }^{3}\left(\frac{\partial \Delta_{\mathrm{S}}}{\partial S}\right)^{3} \\
& \frac{\partial^{4} \omega}{\partial S^{4}} \rightarrow \Delta_{\mathrm{S}}{ }^{8} \frac{\partial^{4} \omega}{\partial S^{4}}+12 \Delta_{\mathrm{S}}{ }^{7} \frac{\partial \Delta_{\mathrm{S}}}{\partial S} \frac{\partial^{3} \omega}{\partial S^{3}}+36 \Delta_{\mathrm{S}}{ }^{6}\left(\frac{\partial \Delta_{\mathrm{S}}}{\partial S}\right)^{2} \frac{\partial^{2} \omega}{\partial S^{2}} \\
& \quad+24 \Delta_{\mathrm{S}}{ }^{5}\left(\frac{\partial \Delta_{\mathrm{S}}}{\partial S}\right)^{3} \frac{\partial \omega}{\partial S}-12 \gamma \Delta_{\mathrm{S}}{ }^{4}\left(\frac{\partial \Delta_{\mathrm{S}}}{\partial S}\right)^{4}
\end{aligned}
$$

\footnotetext{
${ }^{5}$ It is worth pointing out that there is another non-holomorphic quantity that depends only on the periods that is invariant under dilatations and $\mathrm{U}(1)$ transformations, namely the metric

$$
N_{I J}(X, \bar{X}) \equiv 2 \operatorname{Im}\left[F_{I J}(X)\right] .
$$

A special feature of the STU-model is that $\ln \left|\operatorname{det} N_{I J}\right|$ satisfies a similar limit as $\mathcal{K}$, namely

$$
\ln \left|\operatorname{det} N_{I J}\right|=2 \ln [(S+\bar{S})(T+\bar{T})(U+\bar{U})]+\mathcal{O}(\Omega) .
$$


The presence of the derivatives on $\Delta(S)$ on the right-hand side indicates that we are not dealing with covariant quantities. Therefore we explicitly modify the derivatives on the lefthand side to eliminate these non-covariant variations, and denote the resulting covariant expressions by $I^{(2)}(S), I^{(3)}(S)$ and $I^{(4)}(S)$, which will transform as

$$
I^{(n)}(S) \rightarrow I^{(n)}\left(S^{\prime}\right)=\Delta_{\mathrm{S}}(S)^{2 n} I^{(n)}(S) .
$$

The explicit expressions are

$$
\begin{aligned}
I^{(2)}(S) & =\frac{\partial^{2} \omega}{\partial S^{2}}+\frac{1}{2 \gamma}\left(\frac{\partial \omega}{\partial S}\right)^{2} \\
I^{(3)}(S) & =\frac{\partial^{3} \omega}{\partial S^{3}}+\frac{3}{\gamma} \frac{\partial^{2} \omega}{\partial S^{2}} \frac{\partial \omega}{\partial S}+\frac{1}{\gamma^{2}}\left(\frac{\partial \omega}{\partial S}\right)^{3} \\
I^{(4)}(S) & =\frac{\partial^{4} \omega}{\partial S^{4}}+\frac{6}{\gamma} \frac{\partial^{3} \omega}{\partial S^{3}} \frac{\partial \omega}{\partial S}+\frac{3}{\gamma}\left(\frac{\partial^{2} \omega}{\partial S^{2}}\right)^{2}+\frac{12}{\gamma^{2}} \frac{\partial^{2} \omega}{\partial S^{2}}\left(\frac{\partial \omega}{\partial S}\right)^{2}+\frac{3}{\gamma^{3}}\left(\frac{\partial \omega}{\partial S}\right)^{4} .
\end{aligned}
$$

Because products of the $I^{(n)}(S)$ will also transform covariantly, the explicit expressions $I^{(n)}$ with $n>3$ are in principle ambiguous. For the expressions above we made sure that the relation

$$
I^{(n+1)}(S)=\mathcal{D}_{S} I^{(n)}(S),
$$

holds, by making a specific choice for $I^{(4)}$. Here $\mathcal{D}_{S}$ denotes a holomorphic covariant derivative, known as the Serre derivative (for more details, see [23]), which acts on $I^{(n)}$ as

$$
\mathcal{D}_{S} I^{(n)}(S) \equiv\left(\frac{\partial}{\partial S}+\frac{n}{\gamma} \frac{\partial \omega(S)}{\partial S}\right) I^{(n)}(S) .
$$

Henceforth we will assume that (2.27) will extend to all integer values of $n \geq 2$, so as to provide a unique basis for all the covariant expressions as polynomials in terms of the $I^{(n)}(S)$. We note that the $I^{(n)}$ can be expressed in terms of linear combinations of products of Eisenstein series of $\Gamma_{0}(2)[24]$,

$$
I^{(n)}=\sum_{k+2 l=n, k \geq 0, l \geq 1} a_{k, l}\left(\tilde{\mathcal{E}}_{2}\right)^{k}\left(\mathcal{E}_{4}\right)^{l},
$$

with real positive constants $a_{k, l}$. Here, $\mathcal{E}_{4}$ is a normalized Eisenstein series of weight 4 of $\Gamma_{0}(2)$, while $\tilde{\mathcal{E}}_{2}$ is the modular form of weight 2 of $\Gamma_{0}(2)$ given by [23]

$$
\tilde{\mathcal{E}}_{2}(\tau)=\frac{1}{2}\left(3 \mathcal{E}_{2}(\tau)-E_{2}(\tau)\right)=2 E_{2}(2 \tau)-E_{2}(\tau),
$$

where $\mathcal{E}_{2}(\tau), E_{2}(\tau)$ denote the normalized Eisenstein series of weight 2 of $\Gamma_{0}(2)$ and $\mathrm{SL}(2 ; \mathbb{Z})$, respectively.

\section{Higher-order contributions to the Wilsonian action}

Having determined the lowest-order result we can now proceed and determine some higherorder contributions in the solution of (2.13), making use of (2.11). More precisely we will 
present the solutions for $\omega^{(n)}(S, T, U)$ for $n=2,3,4,5$. Before doing so we first present the relevant expansions in terms of a parameter $\lambda$, defined as

$$
\lambda=\frac{A}{\left(X^{0}\right)^{2}} .
$$

The following results then follow straightforwardly from (2.14) and (2.11),

$$
\begin{aligned}
X^{0} \frac{\partial \Omega}{\partial X^{0}} & =A\left[2 \gamma-2 \sum_{n=1}^{\infty} \lambda^{n} n \omega^{(n+1)}(S, T, U)\right] \\
\frac{\partial \Omega}{\partial S} & =A\left[\frac{\partial \omega(S)}{\partial S}+\sum_{n=1}^{\infty} \lambda^{n} \frac{\partial \omega^{(n+1)}(S, T, U)}{\partial S}\right] \\
T \stackrel{\mathrm{S}}{\rightarrow} T^{\prime} & =T+\frac{2}{\Delta_{\mathrm{S}}} \frac{\partial \Delta_{\mathrm{S}}}{\partial S}\left[\lambda \frac{\partial \omega(U)}{\partial U}+\sum_{n=2}^{\infty} \lambda^{n} \frac{\partial \omega^{(n)}(S, T, U)}{\partial U}\right],
\end{aligned}
$$

where the last equation specifies the variation of $T$ under S-duality. The S-duality transformation of $U$ follows from this equation upon interchanging $T \leftrightarrow U$, whereas the S-duality transformation of the fields $S$ and $X^{0}$ do not take the form of power series, as is shown in (2.11). The transformations under T- and U-duality follow from triality.

The above expansions can now be substituted into the four equations (2.13). First we consider the first three equations, where the third equation has been simplified by making use of the fourth equation to remove the term proportional to $(\partial \Omega / \partial T)(\partial \Omega / \partial U)$,

$$
\begin{aligned}
& \left(\frac{\partial \omega(T)}{\partial T}\right)_{\mathrm{S}}^{\prime}-\frac{\partial \omega(T)}{\partial T}+\sum_{n=1}^{\infty} \lambda^{n}\left[\frac{1}{\Delta_{\mathrm{S}}^{2 n}}\left(\frac{\partial \omega^{(n+1)}}{\partial T}\right)_{\mathrm{S}}^{\prime}-\frac{\partial \omega^{(n+1)}}{\partial T}\right]=0 \\
& \left(\frac{\partial \omega(U)}{\partial U}\right)_{\mathrm{S}}^{\prime}-\frac{\partial \omega(U)}{\partial U}+\sum_{n=1}^{\infty} \lambda^{n}\left[\frac{1}{\Delta_{\mathrm{S}}^{2 n}}\left(\frac{\partial \omega^{(n+1)}}{\partial U}\right)_{\mathrm{S}}^{\prime}-\frac{\partial \omega^{(n+1)}}{\partial U}\right]=0 \\
& 2 \gamma \frac{\partial \log \Delta_{\mathrm{S}}}{\partial S}+\frac{1}{\Delta_{\mathrm{S}}^{2}}\left(\frac{\partial \omega(S)}{\partial S}\right)_{\mathrm{S}}^{\prime}-\frac{\partial \omega(S)}{\partial S} \\
& +\sum_{n=1}^{\infty} \lambda^{n}\left[\frac{1}{\Delta_{\mathrm{S}}^{2 n+2}}\left(\frac{\partial \omega^{(n+1)}}{\partial S}\right)_{\mathrm{S}}^{\prime}-\frac{\partial \omega^{(n+1)}}{\partial S}-n \frac{\partial \log \Delta_{\mathrm{S}}}{\partial S}\left[\frac{1}{\Delta_{\mathrm{S}}{ }^{2 n}}\left(\omega^{(n+1)}\right)_{\mathrm{S}}^{\prime}+\omega^{(n+1)}\right]\right]=0
\end{aligned}
$$

The above equations should hold for arbitrary values of $\lambda$. Furthermore we remind the reader that the expressions with a prime attached depend on the transformed fields $S^{\prime}$, $T^{\prime}$ and $U^{\prime}$. Upon Taylor expanding in powers of $T^{\prime}-T$ and $U^{\prime}-U$, one will generate additional terms proportional to powers of $\lambda$. The definition of $S^{\prime}$ under S-duality does not involve the parameter $\lambda$ and can be effected directly (for instance, by using equations such as (2.24)). The last equation of (2.13) is more complicated as it involves a double sum,

$$
\begin{aligned}
& \sum_{n=1}^{\infty} n \lambda^{n}\left[\frac{1}{\Delta_{\mathrm{S}}^{2 n}}\left(\omega^{(n+1)}\right)_{\mathrm{S}}^{\prime}-\omega^{(n+1)}\right]+2 \frac{\partial \log \Delta_{\mathrm{S}}}{\partial S} \lambda \\
& \quad \times\left[\frac{\partial \omega(T)}{\partial T}+\sum_{p=1}^{\infty} \lambda^{p} \frac{\partial \omega^{(p+1)}(S, T, U)}{\partial T}\right]\left[\frac{\partial \omega(U)}{\partial U}+\sum_{q=1}^{\infty} \lambda^{q} \frac{\partial \omega^{(q+1)}(S, T, U)}{\partial U}\right]=0 .
\end{aligned}
$$


Note that there are additional equations associated with T- and U-duality. Those follow immediately by applying triality to the ones specified above.

We note that the terms of order $\lambda^{0}$ cancel by virtue of the first equation (2.24). Furthermore, at order $\lambda$, one directly derives the form of $\omega^{(2)}$ from (3.6),

$$
\omega^{(2)}(S, T, U)=\frac{1}{\gamma} \frac{\partial \omega}{\partial S} \frac{\partial \omega}{\partial T} \frac{\partial \omega}{\partial U}
$$

This result is consistent with triality and it satisfies all the other equations (3.3)-(3.5). ${ }^{6}$

Before continuing let us first note the systematics of the results that gradually appears when working out all the variations. The power of $\gamma$ that appear in the various terms of $\omega^{(n)}$ must be equal to $n-k$, where $k$ is the number of functions $\omega$ that are present. Furthermore every contributions must contain precisely $n-1$ derivatives with respect to $S, n-1$ with respect to $T$ and $n-1$ with respect to $U$. Finally $\omega^{(n)}$ will be multiplied by $\lambda^{n-1}$ in the expansion (2.14). This pattern will persist in all the higher-order terms, something that can be deduced from analyzing the original equations.

Let us now turn to the determination of the function $\omega^{(3)}$, starting again with equation (3.6) and collecting all terms proportional to $\lambda^{2}$. This requires to express the term $\omega^{(2)}\left(S^{\prime}, T^{\prime}, U^{\prime}\right)$ to first order in $\lambda$, which yields

$$
\frac{1}{\Delta_{\mathrm{S}}^{2}} \omega^{(2)}\left(S^{\prime}, T^{\prime}, U^{\prime}\right) \rightarrow \frac{2 \lambda}{\gamma} \frac{\partial \Delta_{\mathrm{S}}}{\partial S}\left(\frac{\partial \omega}{\partial S}-2 \gamma \frac{\partial \Delta_{\mathrm{S}}}{\partial S}\right)\left[\frac{\partial^{2} \omega}{\partial T^{2}}\left(\frac{\partial \omega}{\partial U}\right)^{2}+\frac{\partial^{2} \omega}{\partial U^{2}}\left(\frac{\partial \omega}{\partial T}\right)^{2}\right]
$$

Upon inspecting all possible terms contributing to $\Delta_{\mathrm{S}}{ }^{-2}\left(\omega^{(3)}\right)_{\mathrm{S}}^{\prime}-\omega^{(3)}$, one easily verifies that the second derivatives $\partial^{2} \omega / \partial T^{2}$ and $\partial^{2} \omega / \partial U^{2}$ appear, but there is no corresponding variation proportional to $\partial^{2} \omega / \partial S^{2}$. This does not imply that triality will be violated, simply because $\Delta_{S}{ }^{-2}\left(\omega^{(3)}\right)_{S}^{\prime}-\omega^{(3)}$ will vanish for terms that are proportional to the covariant combination $I^{(2)}(S)$ defined in (2.26). Hence one can include a term $I^{(2)}(S) f(T, U)$ into $\omega^{(3)}$, where $f(T, U)$ can be chosen such that the result for $\omega^{(3)}$ becomes consistent with triality. In this way one derives the result

$$
\begin{aligned}
\omega^{(3)}(S, T, U)= & -2 \frac{\partial^{2} \omega}{\partial S^{2}} \frac{\partial^{2} \omega}{\partial T^{2}} \frac{\partial^{2} \omega}{\partial U^{2}} \\
& -\frac{1}{\gamma}\left[\left(\frac{\partial \omega}{\partial S}\right)^{2} \frac{\partial^{2} \omega}{\partial T^{2}} \frac{\partial^{2} \omega}{\partial U^{2}}+\frac{\partial^{2} \omega}{\partial S^{2}}\left(\frac{\partial \omega}{\partial T}\right)^{2} \frac{\partial^{2} \omega}{\partial U^{2}}+\frac{\partial^{2} \omega}{\partial S^{2}} \frac{\partial^{2} \omega}{\partial T^{2}}\left(\frac{\partial \omega}{\partial U}\right)^{2}\right] \\
& +a_{3}\left[\frac{\partial^{2} \omega}{\partial S^{2}}+\frac{1}{2 \gamma}\left(\frac{\partial \omega}{\partial S}\right)^{2}\right]\left[\frac{\partial^{2} \omega}{\partial T^{2}}+\frac{1}{2 \gamma}\left(\frac{\partial \omega}{\partial T}\right)^{2}\right]\left[\frac{\partial^{2} \omega}{\partial U^{2}}+\frac{1}{2 \gamma}\left(\frac{\partial \omega}{\partial U}\right)^{2}\right] .
\end{aligned}
$$

Observe that we have included also an STU-covariant term that is invariant under triality with an undetermined coefficient $a_{3}$. As it turns out this coefficient will only be determined

\footnotetext{
${ }^{6}$ The expression of $\omega^{(2)}(S, T, U)$ is unique. We have verified that it is not possible to add to it a covariant function $g(S, T, U)$ that is also triality invariant. Such a function would lead to a modification of $\omega^{(3)}(S, T, U)$ which would be incompatible with the transformation laws (3.3)-(3.5) and (3.6). We expect that a similar finding applies to all the higher $\omega^{(n+1)}(S, T, U)$ with $n \geq 2$.
} 
at the next order by requiring that $\omega^{(4)}$ will be triality invariant. We stress once more that derivatives of the function $\omega$ can only depend on a single variable, $S, T$ or $U$. The same conclusion holds for the other equations (3.3)-(3.5) where the extra term proportional to $a_{3}$ does not contribute either. For the first two equations this is obvious and for the third one must make use of the fact that

$$
\left(\frac{\partial I^{(2)}(S)}{\partial S}\right)_{\mathrm{S}}^{\prime}=\Delta_{\mathrm{S}}{ }^{6}\left(\frac{\partial I^{(2)}(S)}{\partial S}+4 \frac{\partial \log \Delta_{\mathrm{S}}}{\partial S} I^{(2)}(S)\right) .
$$

Let us now continue the analysis to order $\lambda^{3}$ and consider $\omega^{(4)}$. Following the same steps we find the following expression for $\omega^{(4)}$ that is required by S-duality, without insisting on triality,

$$
\begin{aligned}
\omega^{(4)}= & -\frac{2}{\gamma} \frac{\partial \omega}{\partial S} \frac{\partial^{2} \omega}{\partial S^{2}}\left[\frac{\partial^{3} \omega}{\partial T^{3}} \frac{\partial^{2} \omega}{\partial U^{2}} \frac{\partial \omega}{\partial U}+\frac{1}{2 \gamma} \frac{\partial^{3} \omega}{\partial T^{3}}\left(\frac{\partial \omega}{\partial U}\right)^{3}+\frac{1}{\gamma} \frac{\partial^{2} \omega}{\partial T^{2}} \frac{\partial \omega}{\partial T} \frac{\partial^{2} \omega}{\partial U^{2}} \frac{\partial \omega}{\partial U}+T \leftrightarrow U\right] \\
& -\frac{1}{\gamma^{2}}\left(\frac{\partial \omega}{\partial S}\right)^{3}\left[\frac{\partial^{3} \omega}{\partial T^{3}} \frac{\partial^{2} \omega}{\partial U^{2}} \frac{\partial \omega}{\partial U}+\frac{1}{3} \frac{\partial^{3} \omega}{\partial T^{3}}\left(\frac{\partial \omega}{\partial U}\right)^{3}+T \leftrightarrow U\right] \\
& -\frac{1}{\gamma^{3}}\left(\frac{\partial \omega}{\partial S}\right)^{3} \frac{\partial^{2} \omega}{\partial T^{2}} \frac{\partial \omega}{\partial T} \frac{\partial^{2} \omega}{\partial U^{2}} \frac{\partial \omega}{\partial U} \\
& +\frac{a_{3}}{\gamma}\left[\frac{\partial^{2} \omega}{\partial S^{2}} \frac{\partial \omega}{\partial S}+\frac{1}{2 \gamma}\left(\frac{\partial \omega}{\partial S}\right)^{3}\right]\left[\frac{\partial^{2} \omega}{\partial T^{2}} \frac{\partial \omega}{\partial T}+\frac{1}{2 \gamma}\left(\frac{\partial \omega}{\partial T}\right)^{3}\right]\left[\frac{\partial^{3} \omega}{\partial U^{3}}+\frac{1}{\gamma}\left(\frac{\partial \omega}{\partial U}\right)^{3}\right] \\
& +\frac{a_{3}}{\gamma}\left[\frac{\partial^{2} \omega}{\partial S^{2}} \frac{\partial \omega}{\partial S}+\frac{1}{2 \gamma}\left(\frac{\partial \omega}{\partial S}\right)^{3}\right]\left[\frac{\partial^{3} \omega}{\partial T^{3}}+\frac{1}{\gamma}\left(\frac{\partial \omega}{\partial T}\right)^{3}\right]\left[\frac{\partial^{2} \omega}{\partial U^{2}} \frac{\partial \omega}{\partial U}+\frac{1}{2 \gamma}\left(\frac{\partial \omega}{\partial U}\right)^{3}\right]
\end{aligned}
$$

When insisting on triality it turns out that one must choose $a_{3}=2$. For this value of $a_{3}$ it turns out that there is a remarkable number of cancellations in $\omega^{(3)}$, whose final expression takes the form

$$
\begin{aligned}
\omega^{(3)}= & \frac{1}{4 \gamma^{3}}\left(\frac{\partial \omega}{\partial S} \frac{\partial \omega}{\partial T} \frac{\partial \omega}{\partial U}\right)^{2} \\
& +\frac{1}{2 \gamma^{2}}\left[\frac{\partial^{2} \omega}{\partial S^{2}}\left(\frac{\partial \omega}{\partial T} \frac{\partial \omega}{\partial U}\right)^{2}+\frac{\partial^{2} \omega}{\partial T^{2}}\left(\frac{\partial \omega}{\partial U} \frac{\partial \omega}{\partial S}\right)^{2}+\frac{\partial^{2} \omega}{\partial U^{2}}\left(\frac{\partial \omega}{\partial S} \frac{\partial \omega}{\partial T}\right)^{2}\right]
\end{aligned}
$$

One then obtains the following result for $\omega^{(4)}$,

$$
\begin{aligned}
\omega^{(4)}= & \frac{1}{6 \gamma^{5}}\left(\frac{\partial \omega}{\partial S} \frac{\partial \omega}{\partial T} \frac{\partial \omega}{\partial U}\right)^{3} \\
& +\frac{1}{2 \gamma^{4}}\left[\frac{\partial^{2} \omega}{\partial S^{2}} \frac{\partial \omega}{\partial S}\left(\frac{\partial \omega}{\partial T} \frac{\partial \omega}{\partial U}\right)^{3}+\frac{\partial^{2} \omega}{\partial T^{2}} \frac{\partial \omega}{\partial T}\left(\frac{\partial \omega}{\partial U} \frac{\partial \omega}{\partial S}\right)^{3}+\frac{\partial^{2} \omega}{\partial U^{2}} \frac{\partial \omega}{\partial U}\left(\frac{\partial \omega}{\partial S} \frac{\partial \omega}{\partial T}\right)^{3}\right]
\end{aligned}
$$




$$
\begin{gathered}
+\frac{1}{\gamma^{3}}\left[\left(\frac{\partial \omega}{\partial S}\right)^{3} \frac{\partial^{2} \omega}{\partial T^{2}} \frac{\partial \omega}{\partial T} \frac{\partial^{2} \omega}{\partial U^{2}} \frac{\partial \omega}{\partial U}+\left(\frac{\partial \omega}{\partial T}\right)^{3} \frac{\partial^{2} \omega}{\partial U^{2}} \frac{\partial \omega}{\partial U} \frac{\partial^{2} \omega}{\partial S^{2}} \frac{\partial \omega}{\partial S}\right. \\
\left.+\left(\frac{\partial \omega}{\partial U}\right)^{3} \frac{\partial^{2} \omega}{\partial S^{2}} \frac{\partial \omega}{\partial S} \frac{\partial^{2} \omega}{\partial T^{2}} \frac{\partial \omega}{\partial T}\right] \\
+\frac{1}{6 \gamma^{3}}\left[\frac{\partial^{3} \omega}{\partial S^{3}}\left(\frac{\partial \omega}{\partial T} \frac{\partial \omega}{\partial U}\right)^{3}+\frac{\partial^{3} \omega}{\partial T^{3}}\left(\frac{\partial \omega}{\partial U} \frac{\partial \omega}{\partial S}\right)^{3}+\frac{\partial^{3} \omega}{\partial U^{3}}\left(\frac{\partial \omega}{\partial S} \frac{\partial \omega}{\partial T}\right)^{3}\right] \\
+a_{4} \gamma\left[\frac{\partial^{3} \omega}{\partial S^{3}}+\frac{3}{\gamma} \frac{\partial^{2} \omega}{\partial S^{2}} \frac{\partial \omega}{\partial S}+\frac{1}{\gamma^{2}}\left(\frac{\partial \omega}{\partial S}\right)^{3}\right]\left[\frac{\partial^{3} \omega}{\partial T^{3}}+\frac{3}{\gamma} \frac{\partial^{2} \omega}{\partial T^{2}} \frac{\partial \omega}{\partial T}+\frac{1}{\gamma^{2}}\left(\frac{\partial \omega}{\partial T}\right)^{3}\right] \\
\times\left[\frac{\partial^{3} \omega}{\partial U^{3}}+\frac{3}{\gamma} \frac{\partial^{2} \omega}{\partial U^{2}} \frac{\partial \omega}{\partial U}+\frac{1}{\gamma^{2}}\left(\frac{\partial \omega}{\partial U}\right)^{3}\right],
\end{gathered}
$$

where, again, we introduced a new STU-covariant term proportional to the parameter $a_{4}$, which is triality invariant. The value of $a_{4}$ is again expected to be fixed by insisting on triality in the next order.

Finally we consider the terms proportional to $\lambda^{4}$ and concentrate on the solution for $\omega^{(5)}$. Based on S-duality alone and using the result for the $\omega^{(n)}$ with $n<5$, we arrange the result into an expression symmetric under triality, and two sets of remaining terms. The triality symmetric expression, which will constitute the final result, reads as follows (we organise the terms in inverse powers of $\gamma$ ),

$$
\begin{gathered}
\omega^{(5)=\frac{1}{2 \gamma^{4}}[}\left[\frac{\partial^{3} \omega}{\partial S^{3}} \frac{\partial \omega}{\partial S}\left[\left(\frac{\partial \omega}{\partial T}\right)^{2} \frac{\partial^{2} \omega}{\partial T^{2}}\left(\frac{\partial \omega}{\partial U}\right)^{4}+\left(\frac{\partial \omega}{\partial U}\right)^{2} \frac{\partial^{2} \omega}{\partial U^{2}}\left(\frac{\partial \omega}{\partial T}\right)^{4}\right]\right. \\
+\frac{\partial^{3} \omega}{\partial T^{3}} \frac{\partial \omega}{\partial T}\left[\left(\frac{\partial \omega}{\partial U}\right)^{2} \frac{\partial^{2} \omega}{\partial U^{2}}\left(\frac{\partial \omega}{\partial S}\right)^{4}+\left(\frac{\partial \omega}{\partial S}\right)^{2} \frac{\partial^{2} \omega}{\partial S^{2}}\left(\frac{\partial \omega}{\partial U}\right)^{4}\right] \\
\left.+\frac{\partial^{3} \omega}{\partial U^{3}} \frac{\partial \omega}{\partial U}\left[\left(\frac{\partial \omega}{\partial S}\right)^{2} \frac{\partial^{2} \omega}{\partial S^{2}}\left(\frac{\partial \omega}{\partial T}\right)^{4}+\left(\frac{\partial \omega}{\partial T}\right)^{2} \frac{\partial^{2} \omega}{\partial T^{2}}\left(\frac{\partial \omega}{\partial S}\right)^{4}\right]\right] \\
+\frac{4}{\gamma^{4}} \frac{\partial^{2} \omega}{\partial S^{2}}\left(\frac{\partial \omega}{\partial S}\right)^{2} \frac{\partial^{2} \omega}{\partial T^{2}}\left(\frac{\partial \omega}{\partial T}\right)^{2} \frac{\partial^{2} \omega}{\partial U^{2}}\left(\frac{\partial \omega}{\partial U}\right)^{2} \\
+\frac{1}{24 \gamma^{4}}\left[\frac{\partial^{4} \omega}{\partial S^{4}}\left(\frac{\partial \omega}{\partial T}\right)^{4}\left(\frac{\partial \omega}{\partial U}\right)^{4}+\frac{\partial^{4} \omega}{\partial T^{4}}\left(\frac{\partial \omega}{\partial U}\right)^{4}\left(\frac{\partial \omega}{\partial S}\right)^{4}+\frac{\partial^{4} \omega}{\partial U^{4}}\left(\frac{\partial \omega}{\partial S}\right)^{4}\left(\frac{\partial \omega}{\partial T}\right)^{4}\right] \\
+\frac{1}{2 \gamma^{4}}\left[\left(\frac{\partial \omega}{\partial S}\right)^{4}\left[\left(\frac{\partial^{2} \omega}{\partial T^{2}}\right)^{2} \frac{\partial^{2} \omega}{\partial U^{2}}\left(\frac{\partial \omega}{\partial U}\right)^{2}+\left(\frac{\partial^{2} \omega}{\partial U^{2}}\right)^{2} \frac{\partial^{2} \omega}{\partial T^{2}}\left(\frac{\partial \omega}{\partial T}\right)^{2}\right]\right. \\
+\left(\frac{\partial \omega}{\partial T}\right)^{4}\left[\left(\frac{\partial^{2} \omega}{\partial U^{2}}\right)^{2} \frac{\partial^{2} \omega}{\partial S^{2}}\left(\frac{\partial \omega}{\partial S}\right)^{2}+\left(\frac{\partial^{2} \omega}{\partial S^{2}}\right)^{2} \frac{\partial^{2} \omega}{\partial U^{2}}\left(\frac{\partial \omega}{\partial U}\right)^{2}\right] \\
\left.+\left(\frac{\partial \omega}{\partial U}\right)^{4}\left[\left(\frac{\partial^{2} \omega}{\partial S^{2}}\right)^{2} \frac{\partial^{2} \omega}{\partial T^{2}}\left(\frac{\partial \omega}{\partial T}\right)^{2}+\left(\frac{\partial^{2} \omega}{\partial T^{2}}\right)^{2} \frac{\partial^{2} \omega}{\partial S^{2}}\left(\frac{\partial \omega}{\partial S}\right)^{2}\right]\right]
\end{gathered}
$$




$$
\begin{gathered}
+\frac{1}{4 \gamma^{5}}\left[\left(\frac{\partial \omega}{\partial S}\right)^{4}\left(\frac{\partial \omega}{\partial T}\right)^{4}\left[\left(\frac{\partial^{2} \omega}{\partial U^{2}}\right)^{2}+\frac{\partial^{3} \omega}{\partial U^{3}} \frac{\partial \omega}{\partial U}\right]+\left(\frac{\partial \omega}{\partial T}\right)^{4}\left(\frac{\partial \omega}{\partial U}\right)^{4}\left[\left(\frac{\partial^{2} \omega}{\partial S^{2}}\right)^{2}+\frac{\partial^{3} \omega}{\partial S^{3}} \frac{\partial \omega}{\partial S}\right]\right. \\
\left.+\left(\frac{\partial \omega}{\partial U}\right)^{4}\left(\frac{\partial \omega}{\partial S}\right)^{4}\left[\left(\frac{\partial^{2} \omega}{\partial T^{2}}\right)^{2}+\frac{\partial^{3} \omega}{\partial T^{3}} \frac{\partial \omega}{\partial T}\right]\right] \\
+\frac{2}{\gamma^{5}}\left[\frac{\partial^{2} \omega}{\partial S^{2}}\left(\frac{\partial \omega}{\partial S}\right)^{2}\left(\frac{\partial \omega}{\partial T}\right)^{4} \frac{\partial^{2} \omega}{\partial U^{2}}\left(\frac{\partial \omega}{\partial U}\right)^{2}+\frac{\partial^{2} \omega}{\partial T^{2}}\left(\frac{\partial \omega}{\partial T}\right)^{2}\left(\frac{\partial \omega}{\partial U}\right)^{4} \frac{\partial^{2} \omega}{\partial S^{2}}\left(\frac{\partial \omega}{\partial S}\right)^{2}\right. \\
\left.+\frac{\partial^{2} \omega}{\partial U^{2}}\left(\frac{\partial \omega}{\partial U}\right)^{2}\left(\frac{\partial \omega}{\partial S}\right)^{4} \frac{\partial^{2} \omega}{\partial T^{2}}\left(\frac{\partial \omega}{\partial T}\right)^{2}\right] \\
+\frac{5}{8 \gamma^{6}}\left[\frac{\partial^{2} \omega}{\partial S^{2}}\left(\frac{\partial \omega}{\partial S}\right)^{2}\left(\frac{\partial \omega}{\partial T}\right)^{4}\left(\frac{\partial \omega}{\partial U}\right)^{4}+\frac{\partial^{2} \omega}{\partial T^{2}}\left(\frac{\partial \omega}{\partial T}\right)^{2}\left(\frac{\partial \omega}{\partial U}\right)^{4}\left(\frac{\partial \omega}{\partial S}\right)^{4}\right. \\
\left.+\frac{\partial^{2} \omega}{\partial U^{2}}\left(\frac{\partial \omega}{\partial U}\right)^{2}\left(\frac{\partial \omega}{\partial S}\right)^{4}\left(\frac{\partial \omega}{\partial T}\right)^{4}\right] \\
+\frac{5}{32 \gamma^{7}}\left(\frac{\partial \omega}{\partial S}\right)^{4}\left(\frac{\partial \omega}{\partial T}\right)^{4}\left(\frac{\partial \omega}{\partial U}\right)^{4} \cdot
\end{gathered}
$$

In addition there are two more contributions. One takes the form

$$
\begin{aligned}
{\left[\omega^{(5)}\right]_{1}=} & -\frac{1}{2 \gamma^{4}}\left(I^{(2)}(S)\right)^{2}\left[\frac{\partial^{2} \omega}{\partial T^{2}}\left(\frac{\partial \omega}{\partial T}\right)^{2}\left(\frac{\partial \omega}{\partial U}\right)^{4}+\frac{\partial^{2} \omega}{\partial U^{2}}\left(\frac{\partial \omega}{\partial U}\right)^{2}\left(\frac{\partial \omega}{\partial T}\right)^{4}\right] \\
& -\frac{1}{4 \gamma^{5}}\left(I^{(2)}(S)\right)^{2}\left(\frac{\partial \omega}{\partial T}\right)^{4}\left(\frac{\partial \omega}{\partial U}\right)^{4}-\frac{1}{24 \gamma^{4}} I^{(4)}(S)\left(\frac{\partial \omega}{\partial T}\right)^{4}\left(\frac{\partial \omega}{\partial U}\right)^{4}
\end{aligned}
$$

and is proportional to the S-covariant terms $\left[I^{(2)}(S)\right]^{2}$ and $I^{(4)}(S)$. These terms are not fixed by the equation for $\omega^{(5)}$. The other contribution contains terms that are proportional to the undetermined constant $a_{4}$ introduced in (3.13). They take the form

$$
\begin{aligned}
{\left[\omega^{(5)}\right]_{2}=\frac{a_{4}}{\gamma}[} & \left.\frac{\partial^{3} \omega}{\partial S^{3}}+\frac{3}{\gamma} \frac{\partial^{2} \omega}{\partial S^{2}} \frac{\partial \omega}{\partial S}+\frac{1}{\gamma^{2}}\left(\frac{\partial \omega}{\partial S}\right)^{3}\right] \frac{\partial \omega}{\partial S} \\
& \times\left[\frac{\partial^{4} \omega}{\partial T^{4}}+\frac{3}{\gamma} \frac{\partial^{3} \omega}{\partial T^{3}} \frac{\partial \omega}{\partial T}+\frac{3}{\gamma}\left(\frac{\partial^{2} \omega}{\partial T^{2}}\right)^{2}+\frac{3}{\gamma^{2}} \frac{\partial^{2} \omega}{\partial T^{2}}\left(\frac{\partial \omega}{\partial T}\right)^{2}\right] \\
& \times\left[\frac{\partial^{3} \omega}{\partial U^{3}}+\frac{3}{\gamma} \frac{\partial^{2} \omega}{\partial U^{2}} \frac{\partial \omega}{\partial U}+\frac{1}{\gamma^{2}}\left(\frac{\partial \omega}{\partial U}\right)^{3}\right] \frac{\partial \omega}{\partial U}+[T \leftrightarrow U],
\end{aligned}
$$

This expression is not triality invariant, and neither can it be made invariant by including terms of the form $I^{(4)}(S) f(T, U)+\left[I^{(2)}(S)\right]^{2} g(T, U)$. Hence it follows that $a_{4}=0$.

Therefore $\omega^{(5)}$ is given by (3.14), up to STU-covariant terms consisting of triality symmetric products of $I^{(4)}(S)$ or $\left[I^{(2)}(S)\right]^{2}, I^{(4)}(T)$ or $\left[I^{(2)}(T)\right]^{2}$, and $I^{(4)}(U)$ or $\left[I^{(2)}(U)\right]^{2}$. There are precisely four such terms, multiplied by arbitrary constants and integer powers of $\gamma$ ranging between $\gamma^{-1}$ and $\gamma^{2}$. Based on the experience for $n \leq 4$ so far, we expect that these undetermined terms will be fixed by proceeding with the present analysis to 
level $\lambda^{5}$. However, in the next section we will change strategy, and at the end of section 6 we will then discover an alternative way of proving that $\omega^{(5)}$ will precisely be given by equation (3.14).

Hence at this point we have determined the coefficient functions $\omega^{(n)}$ for $n \leq 5$. We are not aware of any impediment when continuing the present calculation to higher orders and expect that the function $\Omega$ can be uniquely determined from STU-duality combined with triality.

\section{The topological string partition function}

It is possible to obtain a corresponding version of the topological partition functions from the function (2.6) that encodes the Wilsonian effective action. This relation involves a Legendre transform and as a result the topological string will behave differently under the duality symmetries. The transformation rules of its moduli will not be affected by the possible introduction of deformations, such as those associated with $\Omega$. Upon performing the Legendre transform, one obtains the so-called Hesse potential [25], which decomposes into an infinite variety of different functions. One of these functions exhibits the characteristic features of the topological string partition function. As a result the moduli of the topological string are not identical to the moduli that appear in the Wilsonian action. Actually the same phenomenon is encountered in field theory when considering the Lagrangian and the Hamiltonian of a four-dimensional theory with abelian vector gauge fields. The dynamical variables that appear in the Lagrangian are different from those that appear in the Hamiltonian, and the consequences of electric-magnetic duality will be realized in a different way.

The Hesse potential is a real function of the moduli. As we will see, its moduli will transform covariantly under the dualities and the transformation rules do not change because of the presence of possible deformations. Hence the Hesse potential will transform as a function under general duality transformations, which in the model at hand constitute the group $\operatorname{Sp}(8 ; \mathbb{R})$, and it will remain invariant under the subgroup thereof equal to $\Gamma_{0}(2)_{\mathrm{S}} \times \Gamma_{0}(2)_{\mathrm{T}} \times \Gamma_{0}(2)_{\mathrm{U}}$. As was demonstrated in [9], the Hesse potential can be obtained from the function $F(X, A)$ that encodes the Wilsonian action. To construct the Hesse potential it is important that the deformation $\Omega$ is real, whereas in (2.6) it we assumed to depend only on the holomorphic moduli. To make $\Omega$ real we will simply add its complex conjugate. This change is not problematic as the holomorphic derivatives $F_{I}$ are not affected, so that the results of the previous section will remain valid. When deriving the expression for the Hesse potential, we will for the moment replace $\Omega$ by $\Omega(X, A)+\bar{\Omega}(\bar{X}, \bar{A})$.

The Legendre transformation is most easily understood by first considering a conversion to real special geometry, where the real fields $\left(\phi^{I}, \chi_{J}\right)$ transform under the dualities precisely as the dual pair $\left(X^{I}, F_{J}(X, A)\right)$, where $F_{J}(X, A)$ denotes the derivatives of the function (2.6) with respect to the $X^{J}$. Hence we consider the redefinitions,

$$
\phi^{I}=2 \operatorname{Re} X^{I}, \quad \chi_{J}=2 \operatorname{Re} F_{J}(X, A) .
$$


The Hesse potential is now obtained by a Legendre transform with respect to the imaginary part of the $X^{I}[25]$,

$$
\mathcal{H}(\phi, \chi)=4[\operatorname{Im} F(X)+\Omega(X, A)+\bar{\Omega}(\bar{X}, \bar{A})]+\mathrm{i} \chi_{I}\left(X^{I}-\bar{X}^{I}\right),
$$

where $F(X)$ is equal to the function (2.2). The Hesse potential transforms as a function under generic $\operatorname{Sp}(8 ; \mathbb{R})$ dualities and is left invariant under the $\Gamma_{0}(2)_{\mathrm{S}} \times \Gamma_{0}(2)_{\mathrm{T}} \times \Gamma_{0}(2)_{\mathrm{U}}$ subgroup.

The topological string partition function are conventionally written in terms of complex moduli that transform covariantly under the dualities. Therefore we carry out a conversion by subsequently following the inverse procedure (4.1), but to different moduli $\mathcal{X}^{I}$,

$$
\begin{aligned}
2 \operatorname{Re} X^{I} & =\phi^{I}=2 \operatorname{Re} \mathcal{X}^{I}, \\
2 \operatorname{Re} F_{I}(X, A) & =\chi_{I}=2 \operatorname{Re} \mathcal{F}_{I}(\mathcal{X}),
\end{aligned}
$$

where on the left-hand side we have the original fields $X^{I}$ and the $X^{I}$ - derivatives of the function (2.6), and on the right-hand side the new fields $\mathcal{X}^{I}$ and the $\mathcal{X}^{I}$-derivatives of the classical function $\mathcal{F}(\mathcal{X})$, equal to

$$
\mathcal{F}(\mathcal{X})=-\frac{\mathcal{X}^{1} \mathcal{X}^{2} \mathcal{X}^{3}}{\mathcal{X}^{0}}
$$

This relation is motivated by the fact that both sides of these equations transform consistently under the same duality transformations. Obviously the $X^{I}$ and $\mathcal{X}^{I}$ will differ by terms proportional to powers of $\Omega$. The details of this construction have been described in [9]. The next step is to express the original moduli $X^{I}$ in terms of the new ones, $\mathcal{X}^{I}$; this can be done by iteration. The results can then be substituted into the expression (4.2). Similar evaluations have been carried out in $[7,9]$ for various models. However, to make contact with the topological string partition function it is important to realize that the Hesse potential will decompose into an infinite number of functions that are separately invariant under the action of the duality subgroup that constitute an invariance of the model. The general situation may be described as follows,

$$
\begin{aligned}
\mathcal{H}= & \mathcal{H}^{(0)}+\mathcal{H}^{(1)}+\mathcal{H}^{(2)}+\left(\mathcal{H}_{1}^{(3)}+\mathcal{H}_{2}^{(3)}+\text { h.c. }\right)+\mathcal{H}_{3}^{(3)}+\mathcal{H}_{1}^{(4)}+\mathcal{H}_{2}^{(4)}+\mathcal{H}_{3}^{(4)} \\
& +\left(\mathcal{H}_{4}^{(4)}+\mathcal{H}_{5}^{(4)}+\mathcal{H}_{6}^{(4)}+\mathcal{H}_{7}^{(4)}+\mathcal{H}_{8}^{(4)}+\mathcal{H}_{9}^{(4)}+\text { h.c. }\right) \ldots
\end{aligned}
$$

The leading terms of some of these functions are presented in [9]. For the STU-model of this paper, each of these functions will be invariant under $\left[\Gamma_{0}(2)\right]^{3}$ dualities.

The first function, $\mathcal{H}^{(0)}(\mathcal{X}, \overline{\mathcal{X}})$ in $(4.5)$ is simply the Hesse potential associated with the classical function (4.4), which is real and non-holomorphic,

$$
\mathcal{H}^{(0)}(\mathcal{X}, \overline{\mathcal{X}})=-\mathrm{i}\left[\overline{\mathcal{X}}^{I} \mathcal{F}_{I}(\mathcal{X})-\mathcal{X}^{I} \overline{\mathcal{F}}_{I}(\overline{\mathcal{X}})\right]
$$

As shown in (4.5), there are infinitely many additional functions that emerge which all depend on the extension $\Omega$. For a general real function $\Omega, \mathcal{H}^{(1)}$ has been presented up to terms of order $\Omega^{5}$. However, as was pointed out earlier, for the STU-model the function $\Omega$ 
is actually harmonic, so it can be written as the sum of a holomorphic function $\Omega$ and its complex conjugate. Therefore it suffices to only give the terms proportional to $A$, so that $\Omega$ will depend holomorphically on the modular forms $\omega$,

$$
\begin{aligned}
\mathcal{H}^{(1)}=[4 & \Omega-4 N^{I J} \Omega_{I} \Omega_{J}+8 \Omega_{I J}(N \Omega)^{I}(N \Omega)^{J} \\
& +\frac{8}{3} \mathrm{i} \mathcal{F}_{I J K}(N \Omega)^{I}(N \Omega)^{J}(N \Omega)^{K} \\
& -\frac{4}{3} \mathrm{i}\left(\mathcal{F}_{I J K L}+3 \mathrm{i} \mathcal{F}_{R(I J} N^{R S} \mathcal{F}_{K L) S}\right)(N \Omega)^{I}(N \Omega)^{J}(N \Omega)^{K}(N \Omega)^{L} \\
& -\frac{16}{3} \Omega_{I J K}(N \Omega)^{I}(N \Omega)^{J}(N \Omega)^{K} \\
& -16 \mathrm{i} \mathcal{F}_{I J K} N^{K P} \Omega_{P Q}(N \Omega)^{I}(N \Omega)^{J}(N \Omega)^{Q} \\
& \left.-16(N \Omega)^{P} \Omega_{P Q} N^{Q R} \Omega_{R K}(N \Omega)^{K}+\mathcal{O}\left(\Omega^{5}\right)\right]+ \text { h.c. . }
\end{aligned}
$$

Here we have used the notation $(N \Omega)^{I}=N^{I J} \Omega_{J},(N \bar{\Omega})^{I}=N^{I J} \Omega_{\bar{J}}$, with $N^{I J}$ the inverse of $N_{I J}=2 \operatorname{Im}\left[\mathcal{F}(\mathcal{X})_{I J}\right]$. Explicit expressions for both $N_{I J}$ and $N^{I J}$ are given in appendix A. Note that the whole expression is now written in terms of the new moduli $\mathcal{X}^{I}$, and no longer in terms of the original moduli.

All other functions in (4.5) are qualitatively very different from $\mathcal{H}^{(1)}(\mathcal{X}, \overline{\mathcal{X}})$ : they do not contain terms linear in $\Omega$ and they are not harmonic in $\Omega$. These special features identify the function $\mathcal{H}^{(1)}$ as the unique candidate for the topological string partition function. However, it is important to appreciate that $\mathcal{H}^{(1)}$ is not holomorphic in $\mathcal{X}^{I}$ in view of the presence of the tensors $N^{I J}$. The results of the previous sections based on (2.14) imply that the function $\mathcal{H}^{(1)}$ does instead have the following form,

$$
\mathcal{H}^{(1)}=4 A[-\gamma \ln \lambda+h(\omega ; \lambda)]+\text { h.c. },
$$

where the function $h(\omega ; \lambda)$ depends on the holomorphic modular form $\omega$ and its covariant derivatives, and on the holomorphic topological string coupling constant $\lambda$ defined below. While the modular form $\omega$ depends only on the moduli $S, T, U$, we will see that the covariant derivatives involve a non-holomorphic connection, so that the function $h(\omega ; \lambda)$ will depend explicitly on $S, T, U$, and their complex conjugates. This feature is characteristic for the topological string partition function.

Incidentally, we note that when suppressing all the non-holomorphic terms in (4.7) the function $h(\omega ; \lambda)$ will be holomorphic and only the holomorphic $\Omega$ will remain. In that limit there is no longer a distinction between the old and the new moduli so that $h(\omega ; \lambda)$ must become equal to

$$
h(\omega ; \lambda) \longrightarrow \omega^{(1)}(S, T, U)+\sum_{n=1}^{\infty} \lambda^{n} \omega^{(n+1)}(S, T, U) .
$$

One can use this observation to relate specific terms in (4.7) to the terms in the holomorphic function that was evaluated in section 3 . We will make use of this observation at the end of section 6 and in appendix B. 
Let us now turn to the precise nature to the non-holomorphic terms. First of all, the new moduli and the parameter $\lambda$ are defined by

$$
S=-\mathrm{i} \frac{\mathcal{X}^{1}}{\mathcal{X}^{0}}, \quad T=-\mathrm{i} \frac{\mathcal{X}^{2}}{\mathcal{X}^{0}}, \quad U=-\mathrm{i} \frac{\mathcal{X}^{3}}{\mathcal{X}^{0}} \quad \lambda=\frac{A}{\left(\mathcal{X}^{0}\right)^{2}},
$$

which are similar but not identical to ones defined in previous sections. The fields and $\lambda$ transform under S-duality as

$$
S \rightarrow \frac{a S-\mathrm{i} b}{d+\mathrm{i} c S}, \quad T \rightarrow T, \quad U \rightarrow U, \quad \lambda \rightarrow \frac{\lambda}{(d+\mathrm{i} c S)^{2}},
$$

but one should keep in mind that these fields are fundamentally different from the original ones defined in (2.3), because their duality transformations (4.11) are exact and will not be affected by the presence of $\Omega(X, A)$. As before, the corresponding transformations under T- and U-duality follow from triality.

We explicitly evaluate the first few terms of the function $h(\omega ; \lambda)$ to appreciate how its duality invariance is realized. This follows from repeated use of the following identity, where $V$ and $W$ are two arbitrary functions that depend holomorphically on $\mathcal{X}^{I}$,

$$
\begin{aligned}
\frac{\partial V}{\partial \mathcal{X}^{I}} N^{I J} \frac{\partial W}{\partial \mathcal{X}^{J}}= & \frac{1}{\left(\mathcal{X}^{0}\right)^{2}} \sum_{\text {STU }} \frac{1}{S+\bar{S}} \frac{\partial V}{\partial T} \frac{\partial W}{\partial U} \\
& -\frac{1}{\mathcal{X}^{0}} \sum_{\text {STU }}\left[\frac{\partial V}{\partial \mathcal{X}^{0}} \frac{1}{(S+\bar{S})(T+\bar{T})} \frac{\partial W}{\partial U}+\{V \leftrightarrow W\}\right] \\
& +\frac{2}{(S+\bar{S})(T+\bar{T})(U+\bar{U})} \frac{\partial V}{\partial \mathcal{X}^{0}} \frac{\partial W}{\partial \mathcal{X}^{0}}
\end{aligned}
$$

where we used the explicit expression for the matrix $N^{I J}$ given in (A.3) and where $\sum_{\mathrm{STU}}$ denotes the sum over all independent permutations of $\{S, T, U\}$.

Using the explicit expressions in section 3 , one then obtains the following result for $h(\omega ; \lambda)$,

$$
\begin{gathered}
h(\omega ; \lambda)=\omega(S)+\omega(T)+\omega(U)+\frac{\lambda}{\gamma}\left[D_{S} \omega D_{T} \omega D_{U} \omega\right] \\
+\lambda^{2}\left[\frac{1}{4 \gamma^{3}}\left(D_{S} \omega\right)^{2}\left(D_{T} \omega\right)^{2}\left(D_{U} \omega\right)^{2}\right. \\
+\frac{1}{2 \gamma^{2}}\left[\left(D_{S}^{2} \omega\right)\left(D_{T} \omega\right)^{2}\left(D_{U} \omega\right)^{2}+\left(D_{S} \omega\right)^{2}\left(D_{T}^{2} \omega\right)\left(D_{U} \omega\right)^{2}\right. \\
\left.\left.+\left(D_{S} \omega\right)^{2}\left(D_{T} \omega\right)^{2}\left(D_{U}^{2} \omega\right)\right]\right] \\
+\lambda^{3}\left[\frac{1}{6 \gamma^{5}}\left(D_{S} \omega\right)^{3}\left(D_{T} \omega\right)^{3}\left(D_{U} \omega\right)^{3}\right. \\
+\frac{1}{2 \gamma^{4}}\left[\left(D_{S}^{2} \omega\right)\left(D_{S} \omega\right)\left(D_{T} \omega\right)^{3}\left(D_{U} \omega\right)^{3}\right. \\
+\left(D_{S} \omega\right)^{3}\left(D_{T}^{2} \omega\right)\left(D_{T} \omega\right)\left(D_{U} \omega\right)^{3} \\
\left.+\left(D_{S} \omega\right)^{3}\left(D_{T} \omega\right)^{3}\left(D_{U}^{2} \omega\right)\left(D_{U} \omega\right)\right]
\end{gathered}
$$




$$
\begin{aligned}
& +\frac{1}{\gamma^{3}}\left[\left(D_{S} \omega\right)^{3}\left(D_{T}^{2} \omega\right)\left(D_{T} \omega\right)\left(D_{U}^{2} \omega\right)\left(D_{U} \omega\right)\right. \\
& +\left(D_{S}^{2} \omega\right)\left(D_{S} \omega\right)\left(D_{T}^{2} \omega\right)\left(D_{T} \omega\right)\left(D_{U} \omega\right)^{3} \\
& \left.+\left(D_{S}^{2} \omega\right)\left(D_{S} \omega\right)\left(D_{T} \omega\right)^{3}\left(D_{U}^{2} \omega\right)\left(D_{U} \omega\right)\right] \\
& +\frac{1}{6 \gamma^{3}}\left[\left(D_{S}{ }^{3} \omega\right)\left(D_{T} \omega\right)^{3}\left(D_{U} \omega\right)^{3}+\left(D_{S} \omega\right)^{3}\left(D_{T}{ }^{3} \omega\right)\left(D_{U} \omega\right)^{3}\right. \\
& \left.\left.+\left(D_{S} \omega\right)^{3}\left(D_{T} \omega\right)^{3}\left(D_{U}^{3} \omega\right)\right]\right] \\
& +\mathcal{O}\left(\lambda^{4}\right)
\end{aligned}
$$

where we have introduced non-holomorphic duality covariant derivatives defined such that

$$
D_{S}{ }^{n} \omega \rightarrow \Delta_{\mathrm{S}}{ }^{2 n} D_{S}{ }^{n} \omega
$$

which shows that the expression (4.8) is manifestly STU-duality invariant as it should. Incidentally, the duality transformation of the functions $\omega$ may involve a constant imaginary shift due to the multiplyer system, as discussed in section 2, which will cancel in the variation of (4.21) below. Explicit expressions for the covariant derivatives are, for instance,

$$
D_{S} \omega=\frac{\partial \omega}{\partial S}-\frac{2 \gamma}{S+\bar{S}}, \quad D_{S}^{2} \omega=\frac{\partial^{2} \omega}{\partial S^{2}}+\frac{2}{(S+\bar{S})} \frac{\partial \omega}{\partial S}-\frac{2 \gamma}{(S+\bar{S})^{2}},
$$

while for a covariant quantity $\Sigma(S)$ of weight $p$, which transforms under S-duality according to $\Sigma(S) \rightarrow \Delta_{\mathrm{S}}(S)^{p} \Sigma(S)$, the covariant derivative equals

$$
D_{S} \Sigma(S)=\left(\frac{\partial}{\partial S}+\frac{p}{S+\bar{S}}\right) \Sigma(S)
$$

These results can be combined with similar expressions that involve the Serre derivative. For instance, we note the convenient identity

$$
I^{(n+1)}(S)=D_{S} I^{(n)}(S)+\frac{n}{\gamma} I^{(n)}(S) D_{S} \omega
$$

which follows from (2.27). Another useful identity is,

$$
D_{S}^{2} \omega=I^{(2)}(S)-\frac{1}{2 \gamma}\left(D_{S} \omega\right)^{2}
$$

Let us now return to $h(\omega ; \lambda)$ and rewrite it as follows,

$$
h(\omega ; \lambda)=\omega(S)+\omega(T)+\omega(U)+\sum_{g=2}^{\infty} \lambda^{g-1} F^{(g)}(S, T, U) .
$$

This expression defines the genus expansion of the topological string partition function with $g \geq 2$, where the $F^{(g)}$ depend on the functions $\omega(S), \omega(T)$ and $\omega(U)$ and their covariant 
derivatives, which depend on $S, T, U$ and their complex conjugates. Here $\lambda$ plays the role of the topological string coupling constant. As an example we present the expression for $F^{(2)}$,

$$
F^{(2)}(S, T, U)=\frac{\lambda}{\gamma} D_{S} \omega(S) D_{T} \omega(T) D_{U} \omega(U),
$$

where we stress that the dependence on $\bar{S}, \bar{T}$ and $\bar{U}$ is implicit and contained in the covariant derivatives. For higher genus $g=3,4$ the result can be read off from (4.13). Based on (4.7), we also obtain the genus-1 partition function (which is real and harmonic),

$$
F^{(1)}=-\gamma \ln |\lambda|^{2}+\omega(S)+\omega(T)+\omega(U)+\bar{\omega}(\bar{S})+\bar{\omega}(\bar{T})+\bar{\omega}(\bar{U}),
$$

where here and henceforth we choose $A$ equal to unity.

For $g \geq 2$ the partition functions satisfy a holomorphic anomaly equation of the form

$$
\frac{\partial h}{\partial \bar{S}}=\frac{2 \lambda}{(S+\bar{S})^{2}} D_{T} h D_{U} h
$$

which can be verified up to order $\lambda^{4}$ on the basis of the results obtained so far, with similar equations for the $\bar{T}$ and $\bar{U}$ derivatives. Observe that the anti-holomorphic derivative with respect to $\overline{\mathcal{X}}^{0}$ vanishes, as the dependence on $\lambda$ is holomorphic. The equation (4.22) was encountered in [9] as a result of the diagrammatic structure in the Hesse potential. The same arguments apply in this case, so that we may assume that (4.22) holds to all orders.

Note that the above results do not entirely agree with the holomorphic anomaly equation obtained in $[10,11]$, in particular because $F^{(1)}$ is harmonic and therefore not affected by the anomaly. However, one can replace the term $-\gamma \ln |\lambda|^{2}$ by $-\gamma \ln N$, where $N=\left|\operatorname{det} N_{I J}\right|$, since they transform identically under duality. In that case one obtains

$$
F^{(1)}=-\gamma \ln N+\omega(S)+\omega(T)+\omega(U)+\bar{\omega}(\bar{S})+\bar{\omega}(\bar{T})+\bar{\omega}(\bar{U}) .
$$

Equivalently, one could take the view that we have introduced an extra term equal to $-\gamma\left[\ln N-\ln |\lambda|^{2}\right.$ which is duality invariant and non-harmonic. It seems obvious that this modification will not affect the higher-order terms of $h(\omega ; \lambda)$, because those do already respect the duality invariance.

On the other hand, in [9] we have demonstrated how a non-harmonic term in $F^{(1)}$, which transforms into harmonic variations under duality, will introduce non-harmonic terms into the higher-genus contributions. We have therefore explicitly verified that (4.23) will indeed induce the same non-holomorphic corrections as we have found earlier in (4.13).

\section{Partial determination of the function $h$}

Here and in the next section we will try to further determine the function $h(\omega ; \lambda)$ that comprises the part of the topological string partition function that depends holomorphically on the topological string coupling constant $\lambda$. Inspired by [13], we therefore consider the special limit where the modular forms $\omega$ are suppressed and consider the possibility of an exact expression for the topological string partition function. The expression for the 
function $h$ then takes the form of a power series in terms of an effective coupling constant $\tilde{\lambda}$ defined by

$$
\tilde{\lambda}=\frac{\lambda}{(S+\bar{S})(T+\bar{T})(U+\bar{U})} .
$$

Indeed, suppressing $\omega$ in (4.13) leads to

$$
h_{0}(\tilde{\lambda})=\sum_{n=2} a_{n} \tilde{\lambda}^{n-1}=-8 \gamma^{2} \tilde{\lambda}-32 \gamma^{3} \tilde{\lambda}^{2}+\mathcal{O}\left(\tilde{\lambda}^{3}\right)
$$

where we appended the subscript to indicate that this is only a truncated version of the original function $h(\omega ; \lambda)$. Here we note that $\tilde{\lambda}$ transforms under duality with a phase, e.g. $\tilde{\lambda} \rightarrow\left(\bar{\Delta}_{\mathrm{S}} / \Delta_{\mathrm{S}}\right) \tilde{\lambda}$. However, we will eventually replace $\tilde{\lambda}$ by a modified expansion parameter that is fully STU invariant. The main topic of this section is to determine the exact expression for $h_{0}$ and to derive an equation for the additional terms which will be contained in another function $h_{1}$.

Subsequently we substitute (5.2) into the non-linear equation (4.22). Before doing so we first evaluate the result for $D_{T} h$ and $\partial_{\bar{S}} h$ upon suppressing $\omega$,

$$
\begin{aligned}
\left.(T+\bar{T}) D_{T} h\right|_{\omega=0} & =-2 \gamma+\sum_{n=2}(n-1) a_{n} \tilde{\lambda}^{n-1}, \\
\left.(S+\bar{S}) \frac{\partial h}{\partial \bar{S}}\right|_{\omega=0} & =-\sum_{n=2}(n-1) a_{n} \tilde{\lambda}^{n-1} .
\end{aligned}
$$

Equation (4.22) then leads to $a_{2}=-8 \gamma^{2}$ and $a_{3}=-32 \gamma^{3}$ by considering the terms proportional to $\tilde{\lambda}$ and $\tilde{\lambda}^{2}$, respectively, which is in agreement with the values found in (5.2). The terms in higher powers of $\tilde{\lambda}$ then yield the following equations (for $n \geq 4$ ),

$$
-(n-1) a_{n}=-8 \gamma(n-2) a_{n-1}+2 \sum_{r=2}^{n-2}(r-1)(n-r-1) a_{r} a_{n-r} .
$$

Hence all the coefficients $a_{n}$ will be determined by these equations. It also follows that these coefficients are real. The reader may use this equation to find $a_{4}=-\frac{640}{3} \gamma^{4}$, which can also be directly verified from (4.13). We will need this result shortly.

It is now straightforward to rewrite (4.22) as

$$
\left(\tilde{\lambda} \frac{\partial h_{0}}{\partial \tilde{\lambda}}\right)^{2}+\left(\frac{1}{2 \tilde{\lambda}}-4 \gamma\right) \tilde{\lambda} \frac{\partial h_{0}}{\partial \tilde{\lambda}}+4 \gamma^{2}=0
$$

Since this equation is quadratic we may distinguish two different solutions. One of them reproduces the weak coupling results,

$$
\frac{\mathrm{d} h_{0}(\tilde{\lambda})}{\mathrm{d} \tilde{\lambda}}=\frac{2 \gamma}{\tilde{\lambda}}-\frac{1}{4 \tilde{\lambda}^{2}}[1-\sqrt{1-16 \gamma \tilde{\lambda}}] .
$$

This ordinary differential equation has a solution

$$
h_{0}(\tilde{\lambda})=2 \gamma \ln 4 \gamma \tilde{\lambda}+\frac{1}{4 \tilde{\lambda}}-\frac{\sqrt{1-16 \gamma \tilde{\lambda}}}{4 \tilde{\lambda}}-2 \gamma+4 \gamma \operatorname{arctanh} \sqrt{1-16 \gamma \tilde{\lambda}}
$$


whose expansion in powers of $\tilde{\lambda}$ indeed reproduces the first three terms noted before,

$$
h_{0}(\tilde{\lambda})=-8 \gamma^{2} \tilde{\lambda}-32 \gamma^{3} \tilde{\lambda}^{2}-\frac{640}{3} \gamma^{4} \tilde{\lambda}^{3}-1792 \gamma^{5} \tilde{\lambda}^{4}+\mathcal{O}\left(\tilde{\lambda}^{5}\right) .
$$

Observe that this result for the STU-model is qualitatively different from the result obtained in [13] for general Calabi-Yau compactifications. The function $h_{0}$ constitutes only part of the function $h$ and it is not invariant under the STU-dualities. However, it is straightforward to extend it to a duality invariant function by replacing the definition of $\tilde{\lambda}$ according to

$$
\tilde{\lambda} \longrightarrow \tilde{\lambda}=-\frac{\lambda}{8 \gamma^{3}} D_{S} \omega(S) D_{T} \omega(T) D_{U} \omega(U),
$$

which reduces to the old definition (5.1) when $\omega=0$. Upon this replacement the function $h_{0}(\tilde{\lambda})$ is STU-duality invariant and the first few terms in its expansion are equal to

$$
\begin{aligned}
h_{0}(\tilde{\lambda})= & \sum_{n=2} a_{n} \tilde{\lambda}^{n-1} \\
= & \frac{\lambda}{\gamma}\left[D_{S} \omega(S) D_{T} \omega(T) D_{U} \omega(U)\right]-\frac{\lambda^{2}}{2 \gamma^{3}}\left[D_{S} \omega(S) D_{T} \omega(T) D_{U} \omega(U)\right]^{2} \\
& +\frac{5 \lambda^{3}}{12 \gamma^{5}}\left[D_{S} \omega(S) D_{T} \omega(T) D_{U} \omega(U)\right]^{3} \\
& -\frac{7 \lambda^{4}}{16 \gamma^{7}}\left[D_{S} \omega(S) D_{T} \omega(T) D_{U} \omega(U)\right]^{4}+\mathcal{O}\left(\lambda^{5}\right),
\end{aligned}
$$

which is now manifestly duality invariant. Hence one can decompose $h(\omega ; \lambda)$ as follows,

$$
h(\omega ; \lambda)=\omega(S)+\omega(T)+\omega(U)+h_{0}(\tilde{\lambda})+h_{1}(\tilde{\lambda}),
$$

where the function $h_{1}(\tilde{\lambda})$ should vanish in the limit $\omega=0$, because in that limit $h_{0}$ will already capture all the terms in $h$. Therefore $h_{1}$ can be written in a form that is at least linear in the covariant holomorphic functions $I^{(n)}$ defined in $(2.26)$, or products thereof, which vanish for $\omega=0$, times first order covariant derivatives of the functions $\omega$. Explicit calculations leads to the first few terms in $h_{1}$,

$$
\begin{gathered}
h_{1}(\lambda)=\frac{\lambda^{2}}{2 \gamma^{2}}\left[I^{(2)}(S)\left(D_{T} \omega\right)^{2}\left(D_{U} \omega\right)^{2}+\left(D_{S} \omega\right)^{2} I^{(2)}(T)\left(D_{U} \omega\right)^{2}+\left(D_{S} \omega\right)^{2}\left(D_{T} \omega\right)^{2} I^{(2)}(U)\right] \\
+\frac{\lambda^{3}}{\gamma^{3}}\left[\left(D_{S} \omega\right)^{3} I^{(2)}(T)\left(D_{T} \omega\right) I^{(2)}(U)\left(D_{U} \omega\right)+I^{(2)}(S)\left(D_{S} \omega\right) I^{(2)}(T)\left(D_{T} \omega\right)\left(D_{U} \omega\right)^{3}\right. \\
\left.\quad+I^{(2)}(S)\left(D_{S} \omega\right)\left(D_{T} \omega\right)^{3} I^{(2)}(U)\left(D_{U} \omega\right)\right] \\
+\frac{\lambda^{3}}{\gamma^{4}}\left[I^{(2)}(S)\left(D_{S} \omega\right)\left(D_{T} \omega\right)^{3}\left(D_{U} \omega\right)^{3}+\left(D_{S} \omega\right)^{3} I^{(2)}(T)\left(D_{T} \omega\right)\left(D_{U} \omega\right)^{3}\right. \\
\left.\quad+\left(D_{S} \omega\right)^{3}\left(D_{T} \omega\right)^{3} I^{(2)}(U)\left(D_{U} \omega\right)\right] \\
+\frac{\lambda^{3}}{6 \gamma^{3}}\left[I^{(3)}(S)\left(D_{T} \omega\right)^{3}\left(D_{U} \omega\right)^{3}+\left(D_{S} \omega\right)^{3} I^{(3)}(T)\left(D_{U} \omega\right)^{3}\right. \\
\left.\quad+\left(D_{S} \omega\right)^{3}\left(D_{T} \omega\right)^{3} I^{(3)}(U)\right]+\mathcal{O}\left(\lambda^{4}\right) .
\end{gathered}
$$


It is advantageous to express this result again in the modified coupling constants $\tilde{\lambda}$ defined in (5.9). Because of the invariance under dualities, the expression takes a simpler form,

$$
\begin{aligned}
h_{1}(\tilde{\lambda})= & 32 \gamma^{4}\left(\tilde{\lambda}^{2}+16 \gamma \tilde{\lambda}^{3}\right)\left[\tilde{I}^{(2)}(S)+\tilde{I}^{(2)}(T)+\tilde{I}^{(2)}(U)\right] \\
& -512 \gamma^{6} \tilde{\lambda}^{3}\left[\tilde{I}^{(2)}(T) \tilde{I}^{(2)}(U)+\tilde{I}^{(2)}(S) \tilde{I}^{(2)}(T)+\tilde{I}^{(2)}(S) \tilde{I}^{(2)}(U)\right] \\
& -\frac{256}{3} \gamma^{6} \tilde{\lambda}^{3}\left[\tilde{I}^{(3)}(S)+\tilde{I}^{(3)}(T)+\tilde{I}^{(3)}(U)\right]+\mathcal{O}\left(\tilde{\lambda}^{4}\right)
\end{aligned}
$$

Here we made use of the duality invariant quantities

$$
\tilde{I}^{(n)}(S)=\frac{I^{(n)}(S)}{\left(D_{S} \omega\right)^{n}}
$$

which are no longer holomorphic because of the presence of the non-holomorphic covariant derivative. Hence the function $h_{1}$ can be written in terms of functions of $\tilde{\lambda}$ times polynomials of the $\tilde{I}^{(n)}$ that are invariant under triality.

The function (5.11) must still satisfy the anomaly equation (4.22), and this implies a non-linear differential equation for $h_{1}$. To evaluate this equation we note the relations

$$
\begin{aligned}
\frac{\partial \tilde{\lambda}}{\partial \bar{S}} & =\frac{2 \gamma}{(S+\bar{S})^{2} D_{S} \omega} \tilde{\lambda}, \\
\frac{\partial \tilde{I}^{(n)}(S)}{\partial \bar{S}} & =-\frac{2 n \gamma}{(S+\bar{S})^{2} D_{S} \omega} \tilde{I}^{(n)}(S) \\
D_{T} \tilde{\lambda} & =D_{T} \omega\left(\tilde{I}^{(2)}(T)-\frac{1}{2 \gamma}\right) \tilde{\lambda}, \\
D_{T} \tilde{I}^{(n)}(T) & =D_{T} \omega\left(\tilde{I}^{(n+1)}(T)-\frac{n}{2 \gamma} \tilde{I}^{(n)}(T)-n \tilde{I}^{(n)}(T) \tilde{I}^{(2)}(T)\right),
\end{aligned}
$$

where we made use of (4.17) and (4.18). With the help of these results one derives the equation for $h_{1}(\tilde{\lambda})$,

$$
\begin{aligned}
& \tilde{\lambda} \frac{\partial h_{1}}{\partial \tilde{\lambda}}-\sum_{n=2} n \tilde{I}^{(n)}(S) \frac{\partial h_{1}}{\partial \tilde{I}^{(n)}(S)}= \\
& -16 \gamma^{3} \tilde{\lambda}\left[\tilde{I}^{(2)}(T)-\gamma \tilde{I}^{(2)}(T) \tilde{I}^{(2)}(U)+(T \leftrightarrow U)\right] \\
& -2 \gamma \tilde{\lambda} \frac{\partial h_{0}}{\partial \tilde{\lambda}}\left[(1-4 \gamma \tilde{\lambda}) \tilde{I}^{(2)}(T)-\gamma(1-8 \gamma \tilde{\lambda}) \tilde{I}^{(2)}(T) \tilde{I}^{(2)}(U)+(T \leftrightarrow U)\right] \\
& +4 \gamma \tilde{\lambda}\left[\left(1-\frac{1}{2 \gamma} \tilde{\lambda} \frac{\partial h_{0}}{\partial \tilde{\lambda}}\left(1-2 \gamma \tilde{I}^{(2)}(T)\right)\right)\left(1-2 \gamma \tilde{I}^{(2)}(U)\right)+(T \leftrightarrow U)\right] \tilde{\lambda} \frac{\partial h_{1}}{\partial \tilde{\lambda}} \\
& -8 \gamma^{2} \tilde{\lambda}\left[\left(1-\frac{1}{2 \gamma} \tilde{\lambda} \frac{\partial h_{0}}{\partial \tilde{\lambda}}\left(1-2 \gamma \tilde{I}^{(2)}(T)\right)\right)\right. \\
& \left.\quad \times \sum_{n=2}\left(\tilde{I}^{(n+1)}(U)-\frac{n}{2 \gamma} \tilde{I}^{(n)}(U)-n \tilde{I}^{(n)}(U) \tilde{I}^{(2)}(U)\right) \frac{\partial h_{1}}{\partial \tilde{I}^{(n)}(U)}+(T \leftrightarrow U)\right]
\end{aligned}
$$




$$
\begin{aligned}
& -8 \gamma^{2} \tilde{\lambda} \\
& \times\left[\left(1-2 \gamma \tilde{I}^{(2)}(T)\right) \frac{1}{2 \gamma} \tilde{\lambda} \frac{\partial h_{1}}{\partial \tilde{\lambda}}-\sum_{m=2}\left(\tilde{I}^{(m+1)}(T)-\frac{m}{2 \gamma} \tilde{I}^{(m)}(T)-m \tilde{I}^{(m)}(T) \tilde{I}^{(2)}(T)\right) \frac{\partial h_{1}}{\partial \tilde{I}^{(m)}(T)}\right] \\
& \times\left[\left(1-2 \gamma \tilde{I}^{(2)}(U)\right) \frac{1}{2 \gamma} \tilde{\lambda} \frac{\partial h_{1}}{\partial \tilde{\lambda}}-\sum_{n=2}\left(\tilde{I}^{(n+1)}(U)-\frac{n}{2 \gamma} \tilde{I}^{(n)}(U)-n \tilde{I}^{(n)}(U) \tilde{I}^{(2)}(U)\right) \frac{\partial h_{1}}{\partial \tilde{I}^{(n)}(U)}\right],
\end{aligned}
$$

where we made use of (5.5) without making use of the weak coupling solution (5.7). We will study the function $h_{1}$ in the next section.

At the end of this section we analyze the more general solution of the differential equation (5.5). Because this equation is quadratic, there are two branches,

$$
\frac{\mathrm{d} h_{0}(\tilde{\lambda})}{\mathrm{d} \tilde{\lambda}}=\frac{2 \gamma}{\tilde{\lambda}}-\frac{1}{4 \tilde{\lambda}^{2}}[1 \mp \sqrt{1-16 \gamma \tilde{\lambda}}]
$$

which correspond to the two sheets of the Riemann surface described by the following algebraic curve in $\mathbb{C}^{2}$,

$$
v^{2}=1-16 \gamma \tilde{\lambda}
$$

where $(\tilde{\lambda}, v) \in \mathbb{C}^{2}$. The first sheet is the one that contains $(\tilde{\lambda}, v)=(0,1)$, while the second sheet is the one that contains $(\tilde{\lambda}, v)=(0,-1)$. The weak coupling result derived so far is then recovered by working in the first sheet in the vicinity of $(\tilde{\lambda}, v)=(0,1)$; the associated ordinary differential equation (5.6) leads to the solution (5.7). Instead of working on one or on the other sheet, we may work with a single variable $u$ on the Riemann surface $\mathbb{C}$, which we identify with $v$ on the first sheet, and with $-v$ on the second sheet. In terms of $u$ the function $h_{0}$ takes the following form,

$$
h_{0}(u)=2 \gamma\left(2 \ln \frac{u+1}{2}-\frac{u-1}{u+1}\right),
$$

and covers both sheets. Note that the solution $h_{0}(u)$ contains a logarithmic branch cut starting at $u=-1$, and it vanishes at the zero-coupling point $u=1$. The equation (5.16) for $h_{1}$ depends on $h_{0}$ only through

$$
\tilde{\lambda} \frac{\partial h_{0}}{\partial \tilde{\lambda}}=2 \gamma \frac{u-1}{u+1}, \quad \tilde{\lambda} \frac{\partial h_{1}}{\partial \tilde{\lambda}}=\frac{u^{2}-1}{2 u} \frac{\partial h_{1}}{\partial u},
$$

where the relation between $\tilde{\lambda}$ and the duality invariant effective coupling constant $u$ is given by

$$
\frac{u^{2}-1}{16 \gamma}=-\tilde{\lambda}=\frac{\lambda}{8 \gamma^{3}} D_{S} \omega(S) D_{T} \omega(T) D_{U} \omega(U) .
$$

We now express equation (5.16) in terms of $u$, and we will regard $h_{1}$ as a function of $u$,

$$
\begin{aligned}
& \frac{u^{2}-1}{2 u} \frac{\partial h_{1}}{\partial u}-\sum_{n=2} n \tilde{I}^{(n)}(S) \frac{\partial h_{1}}{\partial \tilde{I}^{(n)}(S)}= \\
& +\gamma^{2}\left(u^{2}-1\right)\left[\tilde{I}^{(2)}(T)-\gamma \tilde{I}^{(2)}(T) \tilde{I}^{(2)}(U)+(T \leftrightarrow U)\right]
\end{aligned}
$$




$$
\begin{aligned}
& -\gamma^{2} \frac{u-1}{u+1}\left[\left(3+u^{2}\right) \tilde{I}^{(2)}(T)-2 \gamma\left(1+u^{2}\right) \tilde{I}^{(2)}(T) \tilde{I}^{(2)}(U)+(T \leftrightarrow U)\right] \\
& -\frac{1}{4}\left(u^{2}-1\right)\left[\left(1-\frac{u-1}{u+1}\left(1-2 \gamma \tilde{I}^{(2)}(T)\right)\right)\left(1-2 \gamma \tilde{I}^{(2)}(U)\right)+(T \leftrightarrow U)\right] \frac{u^{2}-1}{2 u} \frac{\partial h_{1}}{\partial u} \\
& +\frac{1}{2} \gamma\left(u^{2}-1\right)\left[\left(1-\frac{u-1}{u+1}\left(1-2 \gamma \tilde{I}^{(2)}(T)\right)\right)\right. \\
& \left.\quad \times \sum_{n=2}\left(\tilde{I}^{(n+1)}(U)-\frac{n}{2 \gamma} \tilde{I}^{(n)}(U)-n \tilde{I}^{(n)}(U) \tilde{I}^{(2)}(U)\right) \frac{\partial h_{1}}{\partial \tilde{I}^{(n)}(U)}+(T \leftrightarrow U)\right] \\
& +\frac{1}{2} \gamma\left(u^{2}-1\right) \quad\left[\left(1-2 \gamma \tilde{I}^{(2)}(T)\right) \frac{u^{2}-1}{4 \gamma u} \frac{\partial h_{1}}{\partial u}-\sum_{m=2}\left(\tilde{I}^{(m+1)}(T)-\frac{m}{2 \gamma} \tilde{I}^{(m)}(T)-m \tilde{I}^{(m)}(T) \tilde{I}^{(2)}(T)\right) \frac{\partial h_{1}}{\partial \tilde{I}^{(m)}(T)}\right] \\
& \times\left[\left(1-2 \gamma \tilde{I}^{(2)}(U)\right) \frac{u^{2}-1}{4 \gamma u} \frac{\partial h_{1}}{\partial u}-\sum_{n=2}\left(\tilde{I}^{(n+1)}(U)-\frac{n}{2 \gamma} \tilde{I}^{(n)}(U)-n \tilde{I}^{(n)}(U) \tilde{I}^{(2)}(U)\right) \frac{\partial h_{1}}{\partial \tilde{I}^{(n)}(U)}\right]
\end{aligned}
$$

In the next section we will discuss various partial solutions of this equation. Note that we will be encountering three special values for $u$, namely $u=1,0,-1$. The value $u=1$ corresponds to the perturbative point. The significance of the other two points is at present not entirely clear, as for those values the above equations exhibit singularities.

\section{Evaluating contributions contained in $h_{1}$}

In this section we will start the explicit evaluation of a variety of terms that are contained in the function $h_{1}$ by imposing the holomorphic anomaly equation. This means that we will be studying possible solutions of the differential equation (5.22). Since the function $h_{1}$ is decomposed in terms of products of the quantities $\widetilde{I}^{(n)}$ with $u$-dependent coefficients, we can concentrate on specific products and study the consequences of (5.22). As it turns out, contributions that depend on at most two of the moduli are determined by algebraic equations. For the terms that depend on all three moduli, the situation is more complicated since, for a subclass of these terms, one will have to solve differential equations that will necessarily introduce integration constants.

In the last part of this section we will then use the various results of the topological string partition function and investigate its implications for the effective Wilsonian action. Although part of the input of the latter was taken into account when constructing the former, it turns out that the dual approach that we follow here enables not only to demonstrate the mutual consistency of the corresponding results, but it also enables to resolve the ambiguities that were encountered at this stage. For instance, we will be able to fix an integration constant associated with the differential equations that we will be trying to solve.

Let us first start by considering the terms in $h_{1}(u)$ that only depend on $S$. In that case we derive the following equation from (5.22),

$$
\frac{u^{2}-1}{2} \frac{\partial h_{1}}{\partial u}-\sum_{n=2} n \tilde{I}^{(n)}(S) \frac{\partial h_{1}}{\partial \tilde{I}^{(n)}(S)}-\frac{1}{32 \gamma} \frac{\left(u^{2}-1\right)^{3}}{u^{2}}\left(\frac{\partial h_{1}}{\partial u}\right)^{2}=0 .
$$


However, there is a second equation that follows by interchanging $S$ and $T$ in (5.22); here we also suppress all $T$ - and $U$-dependent terms, which leads to a different equation (because the equation (5.22) is not symmetric under the interchange of $S$ and $T$ ),

$$
\begin{aligned}
& 2 \gamma^{2} \frac{(u-1)^{2}}{u+1} \tilde{I}^{(2)}(S) \\
& -\frac{u^{2}-1}{8 u}\left[3+u^{2}-(u-3)(u-1)\left(1-2 \gamma \tilde{I}^{(2)}(S)\right)\right] \frac{\partial h_{1}}{\partial u} \\
& +\left(\gamma(u-1)-\frac{\left(u^{2}-1\right)^{2}}{8 u} \frac{\partial h_{1}}{\partial u}\right) \sum_{n=2}\left[\tilde{I}^{(n+1)}(S)-\frac{n}{2 \gamma} \tilde{I}^{(n)}(S)-n \tilde{I}^{(n)}(S) \tilde{I}^{(2)}(S)\right] \frac{\partial h_{1}}{\partial \tilde{I}^{(n)}(S)} \\
& +\frac{1}{32 \gamma}\left(1-2 \gamma \tilde{I}^{(2)}(S)\right) \frac{\left(u^{2}-1\right)^{3}}{u^{2}}\left(\frac{\partial h_{1}}{\partial u}\right)^{2}=0 .
\end{aligned}
$$

Combining this equation with (6.1), we derive an equation that depends at most linearly on $\partial h_{1} / \partial u$,

$$
\begin{aligned}
& \gamma \tilde{I}^{(2)}(S)\left[2 \gamma \frac{(u-1)^{2}}{u+1}-\frac{\left(u^{2}-1\right)\left(u^{2}+3\right)}{4 u} \frac{\partial h_{1}}{\partial u}\right] \\
& +\left(\gamma(u-1)-\frac{\left(u^{2}-1\right)^{2}}{8 u} \frac{\partial h_{1}}{\partial u}\right) \sum_{n=2} \tilde{I}^{(n+1)}(S) \frac{\partial h_{1}}{\partial \tilde{I}^{(n)}(S)} \\
& -\frac{1}{2}\left(1+2 \gamma \tilde{I}^{(2)}(S)\right)\left(u-1-\frac{\left(u^{2}-1\right)^{2}}{8 \gamma u} \frac{\partial h_{1}}{\partial u}\right) \sum_{n=2} n \tilde{I}^{(n)}(S) \frac{\partial h_{1}}{\partial \tilde{I}^{(n)}(S)} \\
& -\left(1-2 \gamma \tilde{I}^{(2)}(S)\right) \sum_{n=2} n \tilde{I}^{(n)}(S) \frac{\partial h_{1}}{\partial \tilde{I}^{(n)}(S)}=0 .
\end{aligned}
$$

From the above equation one can then straightforwardly derive the contributions to $h_{1}$ that are linearly proportional to $\tilde{I}^{(n)}(S)$. The resulting expression, which also satisfies (6.1), takes the form,

$$
\left.h_{1}(u)\right|_{\text {linear }}=\sum_{m=2} c_{m}(u)\left[\tilde{I}^{(m)}(S)+\tilde{I}^{(m)}(T)+\tilde{I}^{(m)}(U)\right],
$$

with

$$
c_{m}(u)=\frac{(2 \gamma)^{m}}{m !}\left(\frac{u-1}{u+1}\right)^{m} .
$$

Note that we included the $T$ - and $U$-dependent terms in (6.4) to make the result manifestly invariant under triality.

Encouraged by this result, we proceed to determine the coefficient functions of the terms in $h_{1}$ equal to $\tilde{I}^{(m)}(S) \tilde{I}^{(n)}(S)$ as well as $\tilde{I}^{(m)}(S) \tilde{I}^{(n)}(T)$,

$$
\begin{aligned}
\left.h_{1}(u)\right|_{\text {quadratic }}=\sum_{m, n=2} & d_{m, n}(u)\left[\tilde{I}^{(m)}(S) \tilde{I}^{(n)}(S)+\tilde{I}^{(m)}(T) \tilde{I}^{(n)}(T)+\tilde{I}^{(m)}(U) \tilde{I}^{(n)}(U)\right] \\
& \quad+e_{m, n}(u)\left[\tilde{I}^{(m)}(S) \tilde{I}^{(n)}(T)+\tilde{I}^{(m)}(T) \tilde{I}^{(n)}(U)+\tilde{I}^{(m)}(U) \tilde{I}^{(n)}(S)\right],
\end{aligned}
$$


where we included the terms related by triality. Obviously $d_{m, n}(u)$ and $e_{m, n}(u)$ are symmetric in $(m, n)$. The contributions (6.6) will be determined from (5.22), which leads to three different equations. The first one is equal to

$$
\begin{aligned}
& \frac{u^{2}-1}{2} \frac{\partial h_{1}}{\partial u}-\frac{1}{32 \gamma} \frac{\left(u^{2}-1\right)^{3}}{u^{2}}\left(\frac{\partial h_{1}}{\partial u}\right)^{2}\left(1-2 \gamma \tilde{I}^{(2)}(T)\right) \\
& -\sum_{n=2} n \tilde{I}^{(n)}(S) \frac{\partial h_{1}}{\partial \tilde{I}^{(n)}(S)}+\gamma(u-1)(u-3) \frac{u^{2}-1}{4 u} \frac{\partial h_{1}}{\partial u} \tilde{I}^{(2)}(T) \\
& +\frac{1}{2 \gamma}\left[\gamma(u-1)-\frac{\left(u^{2}-1\right)^{2}}{8 u} \frac{\partial h_{1}}{\partial u}\right]\left(1+2 \gamma \tilde{I}^{(2)}(T)\right) \sum_{n=2} n \tilde{I}^{(n)}(T) \frac{\partial h_{1}}{\partial \tilde{I}^{(n)}(T)} \\
& -\left[\gamma(u-1)-\frac{\left(u^{2}-1\right)^{2}}{8 u} \frac{\partial h_{1}}{\partial u}\right] \sum_{n=2} \tilde{I}^{(n+1)}(T) \frac{\partial h_{1}}{\partial \tilde{I}^{(n)}(T)}=0,
\end{aligned}
$$

where we have retained all the terms depending on $S$ and $T$ with the exception of a term linear in $\tilde{I}^{(2)}(T)$. A second equation follows from exchanging $S \leftrightarrow T$ in (5.22), suppressing all the $U$-dependent term. This will lead to equation (6.7) with $S$ and $T$ interchanged. Finally the third equation follows from interchanging $S$ and $U$ in (5.22), and subsequently suppressing all terms that depend on $U$; this equation is symmetric in $S$ and $T$,

$$
\begin{aligned}
& \frac{\left(u^{2}-1\right)}{2} \frac{\partial h_{1}}{\partial u}-2 \gamma^{3} \frac{(u-1)^{3}}{u+1} \tilde{I}^{(2)}(S) \tilde{I}^{(2)}(T) \\
& +\gamma \frac{u^{2}-1}{4 u}\left[(u-3)(u-1)\left(\tilde{I}^{(2)}(S)+\tilde{I}^{(2)}(T)\right)-4 \gamma(u-1)^{2} \tilde{I}^{(2)}(S) \tilde{I}^{(2)}(T)\right] \frac{\partial h_{1}}{\partial u} \\
& -\gamma(u-1) \sum_{n=2}\left[\left[\left(1+\gamma(u-1) \tilde{I}^{(2)}(S)\right) \tilde{I}^{(n+1)}(T) \frac{\partial h_{1}}{\partial \tilde{I}^{(n)}(T)}+(S \leftrightarrow T)\right]\right. \\
& \left.\quad-\frac{1}{2 \gamma}\left[\left(1+\gamma(u-1) \tilde{I}^{(2)}(S)\right)\left(1+2 \gamma \tilde{I}^{(2)}(T)\right) n \tilde{I}^{(n)}(T) \frac{\partial h_{1}}{\partial \tilde{I}^{(n)}(T)}+(S \leftrightarrow T)\right]\right] \\
& -\frac{1}{32 \gamma} \frac{\left(u^{2}-1\right)^{3}}{u^{2}}\left(\frac{\partial h_{1}}{\partial u}\right)^{2}\left(1-2 \gamma \tilde{I}^{(2)}(S)\right)\left(1-2 \gamma \tilde{I}^{(2)}(T)\right) \\
& +\frac{\left(u^{2}-1\right)^{2}}{8 u} \frac{\partial h_{1}}{\partial u} \sum_{n=2}\left[\left[\left(1-2 \gamma \tilde{I}^{(2)}(S)\right) \tilde{I}^{(n+1)}(T) \frac{\partial h_{1}}{\partial \tilde{I}^{(n)}(T)}+(S \leftrightarrow T)\right]\right. \\
& \left.\quad-\frac{1}{2 \gamma}\left[\left(1-2 \gamma \tilde{I}^{(2)}(S)\right)\left(1+2 \gamma \tilde{I}^{(2)}(T)\right) n \tilde{I}^{(n)}(T) \frac{\partial h_{1}}{\partial \tilde{I}^{(n)}(T)}+(S \leftrightarrow T)\right]\right] \\
& -\frac{1}{2} \gamma\left(u^{2}-1\right) \sum_{m, n=2}\left[\tilde{I}^{(m+1)}(S) \frac{\partial h_{1}}{\partial \tilde{I}^{(m)}(S)} \tilde{I}^{(n+1)}(T) \frac{\partial h_{1}}{\partial \tilde{I}^{(n)}(T)}\right. \\
& \quad-\frac{1}{2 \gamma}\left[\left(1+2 \gamma \tilde{I}^{(2)}(T)\right) \tilde{I}^{(m+1)}(S) \frac{\partial h_{1}}{\partial \tilde{I}^{(m)}(S)} n \tilde{I}^{(n)}(T) \frac{\partial h_{1}}{\partial \tilde{I}^{(n)}(T)}+(S \leftrightarrow T)\right] \\
& \left.\quad+\frac{1}{4 \gamma^{2}}\left(1+2 \gamma \tilde{I}^{(2)}(S)\right)\left(1+2 \gamma \tilde{I}^{(2)}(T)\right) m \tilde{I}^{(m)}(S) \frac{\partial h_{1}}{\partial \tilde{I}^{(m)}(S)} n \tilde{I}^{(n)}(T) \frac{\partial h_{1}}{\partial \tilde{I}^{(n)}(T)}\right]=0 .
\end{aligned}
$$

Here we have dropped one term linear in $\left(\tilde{I}^{(2)}(S)+\tilde{I}^{(2)}(T)\right)$, since we are interested in determining the quadratic terms in (6.6). 
When considering only terms of second order in the $\tilde{I}^{(n)}$, the equation (6.7) simplifies to

$$
\begin{aligned}
& \frac{u^{2}-1}{2} \frac{\partial h_{1}}{\partial u}-\frac{1}{32 \gamma} \frac{\left(u^{2}-1\right)^{3}}{u^{2}}\left(\frac{\partial h_{1}}{\partial u}\right)^{2} \\
& -\sum_{n=2}\left[n\left[\tilde{I}^{(n)}(S) \frac{\partial h_{1}}{\partial \tilde{I}^{(n)}(S)}-\frac{1}{2}(u-1) \tilde{I}^{(n)}(T) \frac{\partial h_{1}}{\partial \tilde{I}^{(n)}(T)}\right]+\gamma(u-1) \tilde{I}^{(n+1)}(T) \frac{\partial h_{1}}{\partial \tilde{I}^{(n)}(T)}\right] \\
& =-\gamma(u-1)(u-3) \frac{u^{2}-1}{4 u} \frac{\partial h_{1}}{\partial u} \tilde{I}^{(2)}(T) \\
& \quad+\frac{\left(u^{2}-1\right)^{2}}{16 \gamma u} \frac{\partial h_{1}}{\partial u} \sum_{n=2}\left[n \tilde{I}^{(n)}(T)-2 \gamma \tilde{I}^{(n+1)}(T)\right] \frac{\partial h_{1}}{\partial \tilde{I}^{(n)}(T)} \\
& \quad-\gamma(u-1) \tilde{I}^{(2)}(T) \sum_{n=2} n \tilde{I}^{(n)}(T) \frac{\partial h_{1}}{\partial \tilde{I}^{(n)}(T)} .
\end{aligned}
$$

The terms on the right-hand side of this equation yield all the terms quadratic in $\tilde{I}^{(n)}$ and are determined by (6.4).

Also equation (6.8) can be simplified by suppressing all terms that manifestly lead to third and higher orders in $\tilde{I}^{(n)}$,

$$
\begin{aligned}
& \frac{\left(u^{2}-1\right)}{2} \frac{\partial h_{1}}{\partial u}-\frac{1}{32 \gamma} \frac{\left(u^{2}-1\right)^{3}}{u^{2}}\left(\frac{\partial h_{1}}{\partial u}\right)^{2} \\
& +\frac{1}{2}(u-1) \sum_{n=2} n\left[\tilde{I}^{(n)}(S) \frac{\partial h_{1}}{\partial \tilde{I}^{(n)}(S)}+\tilde{I}^{(n)}(T) \frac{\partial h_{1}}{\partial \tilde{I}^{(n)}(T)}\right] \\
& -\gamma(u-1) \sum_{n=2}\left[\tilde{I}^{(n+1)}(S) \frac{\partial h_{1}}{\partial \tilde{I}^{(n)}(S)}+\tilde{I}^{(n+1)}(T) \frac{\partial h_{1}}{\partial \tilde{I}^{(n)}(T)}\right] \\
& =2 \gamma^{3} \frac{(u-1)^{3}}{u+1} \tilde{I}^{(2)}(S) \tilde{I}^{(2)}(T) \\
& -\gamma \frac{u^{2}-1}{4 u}\left[(u-3)(u-1)\left(\tilde{I}^{(2)}(S)+\tilde{I}^{(2)}(T)\right)\right] \frac{\partial h_{1}}{\partial u} \\
& +\gamma(u-1) \sum_{n=2}\left[\left[\gamma(u-1) \tilde{I}^{(2)}(S) \tilde{I}^{(n+1)}(T) \frac{\partial h_{1}}{\partial \tilde{I}^{(n)}(T)}+(S \leftrightarrow T)\right]\right. \\
& \left.-\frac{1}{2}\left[\left((u-1) \tilde{I}^{(2)}(S)+2 \tilde{I}^{(2)}(T)\right) n \tilde{I}^{(n)}(T) \frac{\partial h_{1}}{\partial \tilde{I}^{(n)}(T)}+(S \leftrightarrow T)\right]\right] \\
& -\frac{\left(u^{2}-1\right)^{2}}{8 u} \frac{\partial h_{1}}{\partial u} \sum_{n=2}\left[\tilde{I}^{(n+1)}(T) \frac{\partial h_{1}}{\partial \tilde{I}^{(n)}(T)}-\frac{1}{2 \gamma} n \tilde{I}^{(n)}(T) \frac{\partial h_{1}}{\partial \tilde{I}^{(n)}(T)}+(S \leftrightarrow T)\right] \\
& +\frac{1}{2} \gamma\left(u^{2}-1\right) \sum_{m, n=2}\left[\tilde{I}^{(m+1)}(S) \frac{\partial h_{1}}{\partial \tilde{I}^{(m)}(S)} \tilde{I}^{(n+1)}(T) \frac{\partial h_{1}}{\partial \tilde{I}^{(n)}(T)}\right. \\
& -\frac{1}{2 \gamma}\left[\tilde{I}^{(m+1)}(S) \frac{\partial h_{1}}{\partial \tilde{I}^{(m)}(S)} n \tilde{I}^{(n)}(T) \frac{\partial h_{1}}{\partial \tilde{I}^{(n)}(T)}+(S \leftrightarrow T)\right] \\
& \left.+\frac{1}{4 \gamma^{2}} m \tilde{I}^{(m)}(S) \frac{\partial h_{1}}{\partial \tilde{I}^{(m)}(S)} n \tilde{I}^{(n)}(T) \frac{\partial h_{1}}{\partial \tilde{I}^{(n)}(T)}\right] .
\end{aligned}
$$


Just as in (6.9), the terms on the right-hand side of this equation yield all the terms quadratic in $\tilde{I}^{(n)}$ and are determined by (6.4).

Note that the first lines of (6.9) and of (6.10) are identical. Hence, by taking the difference between these two equations we obtain an equation that does not contain derivatives of the coefficient functions $d_{m, n}(u)$ and $e_{m, n}(u)$, and that relates them to the coefficient functions $c_{m}(u)$ given in (6.5). By comparing terms with the same powers of $\tilde{I}^{(m)}(S) \tilde{I}^{(n)}(S)$ or $\tilde{I}^{(m)}(S) \tilde{I}^{(n)}(T)$, we determine the explicit form of the coefficient functions $d_{m, n}(u)$ and $e_{m, n}(u)$. Let us illustrate this by focussing on the first two functions $d_{2,2}(u)$ and $e_{2,2}(u)$. For the coefficient function $d_{2,2}(u)$ we obtain the relation

$$
d_{2,2}(u)=\frac{\left(u^{2}-1\right)^{2}}{16 \gamma u(u+1)} c_{2}(u) \dot{c}_{2}(u)-\gamma \frac{(u-1)^{2}(u-3)}{8 u} \dot{c}_{2}(u)-\gamma \frac{u-1}{u+1} c_{2}(u),
$$

where here and henceforth we will use the notation $\dot{c}(u)=\mathrm{d} c(u) / \mathrm{d} u$. Substituting (6.5), we find

$$
d_{2,2}(u)=-(2 \gamma)^{3} \frac{(u+2)}{4 u}\left(\frac{u-1}{u+1}\right)^{4} .
$$

For the coefficient function $e_{2,2}(u)$ we derive the relation

$$
\begin{aligned}
e_{2,2}(u)= & \frac{\left(u^{2}-1\right)^{2}}{8 \gamma u(u+1)} c_{2}(u) \dot{c}_{2}(u)+\frac{u-1)}{2 \gamma} c_{2}(u)^{2}-\frac{\gamma(u-1)^{2}(u-3)}{4 u} \dot{c}_{2}(u) \\
& -2 \gamma \frac{(u-1)^{2}}{u+1} c_{2}(u)+2 \gamma^{3} \frac{(u-1)^{3}}{u+1},
\end{aligned}
$$

which, upon using again (6.5), yields

$$
e_{2,2}(u)=\frac{(2 \gamma)^{3}}{u}\left(\frac{u-1}{u+1}\right)^{3}
$$

It can be verified that these expressions for $d_{2,2}(u)$ and $e_{2,2}(u)$ also solve (6.7).

Proceeding in this manner, we obtain the following expressions for the coefficient functions (with $m, n \geq 2$ ),

$$
\begin{aligned}
& d_{m, n}(u)=-\frac{(2 \gamma)^{m+n-1}}{(m-1) !(n-1) !}\left[\frac{1}{m(m+1)}+\frac{1}{n(n+1)}-\frac{(m+n-2) !}{(m+n) !}+\frac{1}{2 u}\right]\left(\frac{u-1}{u+1}\right)^{m+n} \\
& e_{m, n}(u)=\frac{(2 \gamma)^{m+n-1}}{(m-1) !(n-1) !} \frac{1}{u}\left(\frac{u-1}{u+1}\right)^{m+n-1}
\end{aligned}
$$

Note that these functions exhibit singularities at $u=0$ and $u=-1$.

It is remarkable that the coefficient functions $d_{m, n}(u)$ and $e_{m, n}(u)$ are determined by algebraic means, while we started from a differential equation. This is related to the fact that the equation (5.22) that we are trying to solve, is not symmetric under the interchange of the moduli. To be more precise, when considering the coefficient function belonging to a given product of powers of the symmetric combinations $\left[\tilde{I}^{(n)}(S) \tilde{I}^{(n)}(T) \tilde{I}^{(n)}(U)\right]$, the derivative terms cannot be removed and one has to solve a differential equation, which will lead to integration constants. However, when considering structures that are less 
symmetric, one can eliminate the $u$-derivatives on $h_{1}$ by anti-symmetrizing in two of the moduli and find suitable algebraic equations.

We will demonstrate that differential equations cannot altogether be avoided by discussing two examples, whose results are needed at the end of this section when we will try to match the results of this section to the explicit expression obtained in section 3 for the holomorphic function that encodes the Wilsonian action. The first example concerns a coefficient function denoted by $p(u)$, that can be determined algebraically. It appears in $h_{1}$ as

$$
\begin{aligned}
h_{1}(u)=p(u)\left[\left(\tilde{I}^{(2)}(S)\right)^{2}\left(\tilde{I}^{(2)}(T)+\tilde{I}^{(2)}(U)\right)\right. & +\left(\tilde{I}^{(2)}(T)\right)^{2}\left(\tilde{I}^{(2)}(U)+\tilde{I}^{(2)}(S)\right) \\
& \left.+\left(\tilde{I}^{(2)}(U)\right)^{2}\left(\tilde{I}^{(2)}(S)+\tilde{I}^{(2)}(T)\right)\right]+\cdots .
\end{aligned}
$$

Proceeding as before, we consider (5.22) and subtract from it the same equation with $S$ and $T$ interchanged. The difference between these two equations gives rise to an algebraic equation for $p(u)$,

$$
\begin{aligned}
p(u)= & \frac{\gamma(u-1)^{2}(u-3)}{4 u}\left[\dot{d}_{2,2}-\dot{e}_{2,2}\right]-2 \gamma \frac{(u-1)}{(u+1)} e_{2,2}-\frac{u-1}{4}\left(\frac{u^{2}-1}{2 u} \dot{c}_{2}\right)^{2} \\
& +\frac{(u-1)^{2}(u+1)}{8 \gamma u}\left[-c_{2}\left(\dot{d}_{2,2}-\dot{e}_{2,2}\right)+2 d_{2,2} \dot{c}_{2}+2 \gamma c_{2} \dot{c}_{2}\right]
\end{aligned}
$$

It is easy to see that $p(u)$ is proportional to $(u-1)^{4}$. The leading contributions originate from the terms $\dot{e}_{2,2(u)}$ and $e_{2,2}(u)$ in the first line. They lead to

$$
p(u)=\frac{1}{2} \gamma^{4}(u-1)^{4}+\mathcal{O}\left((u-1)^{5}\right) .
$$

The second example, which does not lead to an algebraic equation, concerns the following terms,

$$
\begin{aligned}
h_{1}(u)= & c_{2}(u)\left[\tilde{I}^{(2)}(S)+\tilde{I}^{(2)}(T)+\tilde{I}^{(2)}(U)\right] \\
& +e_{2,2}(u)\left[\tilde{I}^{(2)}(S) \tilde{I}^{(2)}(T)+\tilde{I}^{(2)}(T) \tilde{I}^{(2)}(U)+\tilde{I}^{(2)}(U) \tilde{I}^{(2)}(S)\right] \\
& +v_{2,2,2}(u) \tilde{I}^{(2)}(S) \tilde{I}^{(2)}(T) \tilde{I}^{(2)}(U)+\cdots .
\end{aligned}
$$

Inserting (6.19) into (5.22) leads to the differential equation,

$$
\begin{aligned}
& \frac{\left(u^{2}-1\right)}{2} \dot{v}_{2,2,2}-2(2-u) v_{2,2,2} \\
& =\frac{\left(u^{2}-1\right)^{2}}{4 u}\left[2 \gamma \dot{e}_{2,2}-4 \gamma \dot{e}_{2,2} \frac{u-1}{u+1}+4 \gamma^{2} \dot{c}_{2}\left(\frac{u-1}{u+1}\right)\right]-2 \gamma e_{2,2}(u-1)^{2} \\
& +\frac{\left(u^{2}-1\right)}{2 \gamma}\left[\frac{\left(u^{2}-1\right)^{2}}{16 u^{2}}\left(6 \dot{e}_{2,2} \dot{c}_{2}-8 \gamma\left(\dot{c}_{2}\right)^{2}\right)\right. \\
& \left.\quad+\frac{\left(u^{2}-1\right)}{2 u}\left(\dot{e}_{2,2} c_{2}+2 e_{2,2} \dot{c}_{2}-2 \gamma \dot{c}_{2} c_{2}\right)+2 e_{2,2} c_{2}\right] .
\end{aligned}
$$


Evaluating the right-hand side of this expression by using the values for $c_{2}(u)$ and $e_{2,2}(u)$ given in (6.5) and (6.12), one obtains

$$
-\frac{4 \gamma^{4}}{u^{4}}\left(\frac{u-1}{u+1}\right)^{4}\left(u^{2}+1\right)\left(3\left(u^{2}-1\right)-16 u\right) .
$$

The differential equation (6.20) for $v_{2,2,2}(u)$ is then solved by

$$
v_{2,2,2}(u)=8 \gamma^{4}\left(\frac{u-1}{u+1}\right)^{2} F(u)
$$

with

$$
F(u)=\frac{1}{(u+1)^{4}}\left[\alpha_{v}-\frac{u^{6}-1-8 u\left(u^{4}+1\right)-3 u^{2}\left(u^{2}-1\right)}{u^{3}}\right],
$$

where $\alpha_{v}$ denotes an integration constant.

At this point we have determined quite a number of coefficient functions in $h_{1}(u)$. To obtain the complete result for the topological string partition function, they have to be combined with the contributions from the function $h_{0}(\tilde{\lambda})$, which was obtained by resummation and is thus known to all orders in $\tilde{\lambda}$. An interesting question is whether one can now re-obtain the result for the holomorphic function that encodes the Wilsonian action, and if so, whether this procedure will have implications for some of the ambiguities that we encountered in section 3 . We will now demonstrate that this is indeed the case.

As we argued in the text above (4.9), one can in principle match the results for the topological string partition function with those for the Wilsonian action. The latter is expressed in terms of the holomorphic functions $\omega^{(n)}$ that, for $n \leq 5$, were determined in section 3. To do so, note that the coefficient functions of $h_{1}(u)$ have to be expressed in terms $\tilde{\lambda}$, which can be achieved by first expressing the duality invariant coupling $u$ in terms of $\tilde{\lambda}$ by an expansion about the perturbative point $u=1$,

$$
u=1-8 \gamma \tilde{\lambda}-32 \gamma^{2} \tilde{\lambda}^{2}+\mathcal{O}\left(\tilde{\lambda}^{3}\right) .
$$

After this conversion one writes $\tilde{\lambda}$ in terms of $\lambda$, the parameter used in section 2, making use of equation (5.9), and substitutes the explicit expression for the quantities $\tilde{I}^{(n)}$. Finally one suppresses all the (non-holomorphic) connections in the covariant derivatives $D \omega$ with respect to the moduli, by replacing $D \omega$ with simple derivatives $\partial \omega$. The result of this truncation can then be compared to the functions $\omega^{(n)}$ with $n \leq 5$. The terms belonging to $\omega^{(n)}$ can then simply be identified because they will be proportional to $\lambda^{(n-1)}$, as follows from the expansion (2.14). We will now briefly review the results of this analysis.

We first note that according to the procedure sketched above, the function $h_{0}$ will take the form of a power series in $\lambda \partial_{S} \omega \partial_{T} \omega \partial_{U} \omega$, where $\lambda$ denotes the original expansion parameter in section 3. In equation (5.10) we have recorded the first four terms of this expansion, where we may now drop the (non-holomorphic) connections in the covariant derivatives. Such terms also appear in the functions $\omega^{(n)}$ that encode the Wilsonian effective action, but it is easy to see that the terms in $\omega^{(2)}-\omega^{(5)}$ do not agree with the first four terms in (5.10). Hence the contributions from $h_{1}(u)$ should compensate for this difference. Indeed, 
such terms are present in $h_{1}(u)$, and they originate from those terms in the quantities $\tilde{I}^{(n)}$ that are proportional to a constant. For convenience we list the values of these constants for $n=2,3,4$, which follow directly from (2.26),

$$
\tilde{I}_{\text {constant }}^{(2)}=\frac{1}{2 \gamma}, \quad \tilde{I}_{\text {constant }}^{(3)}=\frac{1}{\gamma^{2}}, \quad \tilde{I}_{\text {constant }}^{(4)}=\frac{3}{\gamma^{3}} .
$$

Let us now identify the contributions from $h_{1}(u)$ that will contribute to terms that are at most of fourth order in $(u-1)$. They consist of the terms proportional to $c_{2}(u), c_{3}(u)$ and $c_{4}(u)$ in (6.4), the terms proportional to $d_{2,2}(u), e_{2,2}(u)$ and $e_{2,3}(u)$ in (6.6), and finally the terms proportional to the coefficient functions $p(u)$ and $v_{2,2,2}(u)$ that appear in (6.16) and (6.19), respectively.

In principle all these contributions may contribute to the functions $\omega^{(2)}-\omega^{(5)}$ determined in sections 2 and 3. Let us first concentrate on the constant part of the functions $\tilde{I}^{(n)}$ that will combine with the terms of $h_{0}(\tilde{\lambda})$. In that case the relevant contributions from $h_{1}(u)$ will take the form,

$$
\begin{aligned}
\left.h_{1}(u)\right|_{\text {constant }}= & \frac{3}{2 \gamma} c_{2}(u)+\frac{3}{\gamma^{2}} c_{3}(u)+\frac{9}{\gamma^{3}} c_{4}(u)+\frac{3}{4 \gamma^{2}}\left[d_{2,2}(u)+e_{2,2}(u)\right] \\
& +\frac{3}{\gamma^{3}} e_{2,3}(u)+\frac{1}{8 \gamma^{3}}\left[6 p(u)+v_{2,2,2}(u)\right] \cdots .
\end{aligned}
$$

From the explicit expressions of the coefficient functions one can easily determine that only the first term proportional to $c_{2}(u)$ and the term proportional to $v_{2,2,2}(u)$ will contain terms of second order in $(u-1)$, depending on the value of the integration constant $\alpha_{v}$. Upon using (6.24) it follows that the corresponding contribution from $c_{2}(u)$ to $h_{1}(u)$ is equal to $48 \gamma^{3} \tilde{\lambda}^{2}$. There is also a similar contribution from $h_{0}(\tilde{\lambda})$ which, according to (5.8), equals $-32 \gamma^{3} \tilde{\lambda}^{2}$, so that sum of these two contributions yields $16 \gamma^{3} \tilde{\lambda}^{2}$. Converting $\tilde{\lambda}$ to $\lambda$ by using (5.9), and suppressing the connections in the covariant derivative, one reproduces exactly the first term in the expression for $\omega^{(3)}$ given in (3.12). Therefore it follows that the function $F(u)$ must vanish for $u=1$, which fixes the integration constant $\alpha_{v}$ to

$$
\alpha_{v}=-16
$$

With this result $v_{2,2,2}(u)$ reads,

$$
v_{2,2,2}(u)=-\frac{8 \gamma^{4}}{u^{3}}\left(\frac{u-1}{u+1}\right)^{4}\left(u^{2}-1-8 u\right) .
$$

We can now determine all terms in (6.26), so that one can evaluate all the contributions up to order $\tilde{\lambda}^{5}$, leading to

$$
\left.h_{1}(u)\right|_{\text {constant }}=48 \gamma^{3} \tilde{\lambda}^{2}+128 \gamma^{4} \tilde{\lambda}^{3}+2432 \gamma^{5} \tilde{\lambda}^{4}+\mathcal{O}\left(\tilde{\lambda}^{5}\right) .
$$

Adding the contributions from $h_{0}(\tilde{\lambda})$ given in (5.8), and converting $\tilde{\lambda}$ to $\lambda$ as explained above, we precisely reproduce the term $\left(\lambda \partial_{S} \omega \partial_{T} \omega \partial_{U} \omega\right)^{n-1}$ that appears in the expressions for $\omega^{(n)}$ given in (3.12), (3.13) (with $\left.a_{4}=0\right)$ and (3.14). 
Of course, the functions $\tilde{I}^{(n)}$ also contain non-constant terms, which contribute to $h_{1}(u)$. One can verify that they precisely reproduce all the remaining terms in the expressions (3.12), (3.13) (with $a_{4}=0$ ) and (3.14). This was already pointed out at the end of section 3 .

\section{$7 \quad$ Summary and conclusions}

In this paper we studied both the holomorphic function that encodes the Wilsonian effective action of the STU-model of [1], as well as its corresponding topological string partition function. This was done by exploiting the exact duality symmetries of this model and its invariance under arbitrary permutations of its three moduli, $S, T$, and $U$, which is known as triality. The topological string partition function can be obtained from the effective action by carrying out a Legendre transform, as was explained in [9]. Based on preliminary calculations there was the suggestion that the effective action of this STU-model might be exactly calculable by virtue of its high degree of symmetry. The fact that the duality transformations act non-linearly on the function that encodes the Wilsonian action (which itself is not invariant) was seen as another indication that the answer might even be unique. The present paper addresses this and related questions.

A separate motivation for this work was that the connection between the effective action and the topological string had never been worked out explicitly for a realistic model. ${ }^{7}$ Especially in the case that exact results can be obtained, one may be able to obtain valuable insights on this issue. Here we should note that in this paper we only consider the specialKähler moduli that describe the vector supermultiplets.

The initial attempt to explicitly determine the Wilsonian action is described in section 3. Because of supersymmetry the underlying holomorphic function takes the form given in (2.6), where $\Omega$ is decomposed into an infinite set of coefficient functions $\omega^{(n)}(S, T, U)$, as is shown in (2.14). The exact expression in an expansion in terms of the gravitational coupling $\lambda=A /\left(X^{0}\right)^{2}$ is determined by imposing the invariance under dualities and triality up to $\omega^{(5)}$. While this indicates that an exact determination is possible, it is also clear that the strategy adopted here could not easily be continued at arbitrary high orders. Furthermore we should stress that the results for the holomorphic function encode a complete $N=2$ supergravity action, which describes all the terms at the two-derivative level as well as the higher-order couplings to the square of the Weyl tensor.

At this point it is advantageous to direct the attention to the topological string partition function, using the results obtained for the effective action as input. Here one advantage was that the topological string partition function must be invariant under duality. Another important is that the moduli for the topological string transform covariantly and their transformations remain the same at all orders in the genus expansion, unlike the moduli associated with the effective action whose transformations keep changing when proceeding to higher orders.

\footnotetext{
${ }^{7}$ Note that we are not implying that this result equals the unique expression for the topological string partition function of this STU-model, as it is often possible to incorporate additional non-holomorphic terms. This possibility was actually discussed in [9].
} 
The results given in [9] were obtained in the context of iterative expansions, so that the explicit relation with the Wilsonian action was only spelled out for low genus. Here we concentrate on the function $h(\omega ; \lambda)$ that comprises the part of the topological string partition function that depends holomorphically on the topological string coupling constant. By following a similar strategy as in the work of [13], we determine an infinite series of terms by imposing the holomorphic anomaly equation, which can be summed. The resulting function is denoted by $h_{0}(u)$, which depends on an effective duality invariant parameter $u$ that is defined on a Riemann surface $\mathbb{C}$. The explicit form of this function is given in equation (5.19). Unfortunately, it turns out that this result captures only part of the topological string partition function. The reason is that there exist certain holomorphic invariants expressed in terms of ordinary holomorphic derivatives of the modular form $\omega$, which are present in the topological string partition function. These invariants, denoted by $I^{(n)}$, were already introduced at the end of section 2 , and they are functions of either one of the moduli $S, T$, or $U$.

There thus exists an infinite variety of invariants consisting of arbitrary products of $I^{(m)}(S), I^{(n)}(T)$ and $I^{(p)}(U)$, which are multiplied by corresponding coefficient functions that depend on $u$. In principle this does not imply that these functions cannot be determined exactly. In fact we have determined a number of them in section 6 . Specifically, we have determined all terms that are linear and quadratic in the invariants $I^{(n)}$, as well as some of the cubic terms, and we have verified their correctness by comparing their holomorphic contributions to corresponding terms in the effective action. All these invariants have been assigned to a second function that we denoted by $h_{1}(u)$, so that

$$
h(\omega ; \lambda)=\omega(S)+\omega(T)+\omega(U)+h_{0}(u)+h_{1}(u),
$$

where $h_{0}(u)$ was defined in (5.19) and various contributions to $h_{1}(u)$ have been determined in section 6 . While we have presented convincing evidence that also the function $h_{1}(u)$ can be determined exactly, the situation regarding $h_{1}(u)$ remains somewhat unsatisfactory, because we are dealing with an infinite set of coefficient functions.

An interesting observation concerns the behaviour of the coefficient functions in $h_{1}(u)$ as compared to $h_{0}(u)$. The latter function has a logarithmic branch cut starting at $u=-1$ and it is vanishing at $u=1$, as is shown in equation (5.19). The coefficient functions in $h_{1}(u)$ also exhibit higher-order poles at $u=-1$ and zeroes at $u=1$, but in addition they also have poles at $u=0$. So far we have not been able to give an explanation for the presence of these new poles. We note that in the limit where we take the real part of two of the three moduli $S, T, U$ large, all these singularities disappear. In this interesting limit, the form of $\Omega$ greatly simplifies, as we briefly describe in appendix B.

\section{Acknowledgments}

We would like to thank Cristina Câmara, Justin David, Thomas Grimm, Abhiram Kidambi, Suresh Nampuri, Ricardo Schiappa, Ashoke Sen and Stefan Theisen for valuable discussions. We thank the International Center for Theoretical Sciences (ICTS, Bangalore), the Mainz Institute for Theoretical Physics (MITP), the Kavli Institute for the Physics and 
Mathematics of the Universe (IPMU), the International Center for Theoretical Physics (ICTP, Trieste), the Max Planck Institute for Gravitational Physics (AEI, Golm), and the Kavli Institute for Theoretical Physics (KITP) for hospitality during various stages of this work. This research was supported in part by the National Science Foundation under Grant No. NSF PHY-1748958. This work was partially supported by FCT/Portugal through UID/MAT/04459/2019, and by UGC, Govt. of India, through the special assistance programme F-530/13/DRS/2013-2018 (SAP-I).

\section{A The explicit expressions for $N_{I J}$ and $N^{I J}$}

We compute the matrices $N_{I J}$ and $N^{I J}$ for the STU-model based on (2.2). To this end, we display various components of $F_{I J}$,

$$
F_{00}=2 \mathrm{i} S T U, \quad F_{01}=-T U, \quad F_{12}=-\mathrm{i} U .
$$

Then, using $N_{I J}=2 \operatorname{Im} F_{I J}$, we obtain

$$
N_{I J}=\left(\begin{array}{cccc}
2(S T U+\bar{S} \bar{T} \bar{U}) & \mathrm{i}(T U-\bar{T} \bar{U}) \mathrm{i}(U S-\bar{U} \bar{S}) \mathrm{i}(S T-\bar{S} \bar{T}) \\
\mathrm{i}(T U-\bar{T} \bar{U}) & 0 & -(U+\bar{U}) & -(T+\bar{T}) \\
\mathrm{i}(U S-\bar{U} \bar{S}) & -(U+\bar{U}) & 0 & -(S+\bar{S}) \\
\mathrm{i}(T S-\bar{T} \bar{S}) & -(T+\bar{T}) & -(S+\bar{S}) & 0
\end{array}\right)
$$

The inverse matrix $N^{I J}$ reads,

$$
N^{I J}=\mathrm{e}^{\mathcal{K}}\left(\begin{array}{cccc}
2 & \mathrm{i}(S-\bar{S}) & \mathrm{i}(T-\bar{T}) & \mathrm{i}(U-\bar{U}) \\
\mathrm{i}(S-\bar{S}) & 2 S \bar{S} & -(S T+\bar{S} \bar{T}) & -(U S+\bar{U} \bar{S}) \\
\mathrm{i}(T-\bar{T}) & -(S T+\bar{S} \bar{T}) & 2 T \bar{T} & -(T U+\bar{T} \bar{U}) \\
\mathrm{i}(U-\bar{U}) & -(U S+\bar{U} \bar{S}) & -(T U+\bar{T} \bar{U}) & 2 U \bar{U}
\end{array}\right)
$$

where $\operatorname{det} N_{I J}=-\mathrm{e}^{-2 \mathcal{K}}$ and $\mathrm{e}^{-\mathcal{K}}=(S+\bar{S})(T+\bar{T})(U+\bar{U})=-\bar{X}^{I} N_{I J} X^{J}\left|X^{0}\right|^{-2}$.

\section{B The structure of $\Omega$ in the large- $T$ and large- $U$ limit}

In sections 5 and 6 we analyzed the structure of the function $h(\omega ; \lambda)$ that describes part of the topological string partition function. As shown in $(7.1) h(\omega ; \lambda)$ is written in terms of the modular form $\omega$ and two functions $h_{0}(u)$ and $h_{1}(u)$. The function $h_{0}(u)$, given in (5.19), depends on an effective, duality invariant coupling constant $u$ defined in terms of the topological string coupling constant (5.21), and the function $h_{1}(u)$ is decomposed in terms of products of the invariants $\tilde{I}^{(n)}$ with $u$-dependent coefficients that are subject to $(5.22)$.

In this appendix we want to study $h(\omega ; \lambda)$ in the limit where the real part of two of the three moduli $S, T, U$, say $\operatorname{Re} T$ and $\operatorname{Re} U$, are taken to be large. For $\operatorname{Re} T \gg 1, \operatorname{Re} U \gg 1$, the derivatives $\partial_{T} \omega(T)$ and $\partial_{U} \omega(U)$ remain finite, while higher derivatives of $\omega(T)$ and of $\omega(U)$ are exponentially suppressed. As follows directly from (2.26)-(2.28), this leads to

$$
I^{(n)}(T) \approx \frac{(n-1) !}{2 \gamma^{n-1}}\left(\frac{\partial \omega}{\partial T}\right)^{n}
$$


Furthermore the (multiple) non-holomorphic derivatives of $\omega(T)$ satisfy,

$$
D_{T} \omega(T) \approx \partial_{T} \omega(T), \quad D_{T}^{n} \omega(T) \approx 0, \quad(n>1),
$$

as follows from $(4.15)-(4.16)$. As a result the quantities $\tilde{I}^{(n)}$ defined in (5.14), satisfy

$$
\tilde{I}^{(n)}(T) \approx \frac{(n-1) !}{2 \gamma^{n-1}} .
$$

Note that there are identical results for $U$, but not for $S$.

To determine the function $h(\omega ; \lambda)$, we need the functions $h_{0}(u)$ and $h_{1}(u)$. While $h_{0}(u)$ is explicitly known (cf. (5.19)), the function $h_{1}(u)$ must satisfy the non-linear differential equation (5.22), which in the above large-moduli limit simplifies dramatically,

$$
\frac{\left(u^{2}-1\right)}{2 u} \frac{\partial h_{1}}{\partial u}-\sum_{n=2} n \tilde{I}^{(n)}(S) \frac{\partial h_{1}}{\partial \tilde{I}^{(n)}(S)}=\frac{1}{2} \gamma\left(u^{2}-1\right)-2 \gamma \frac{u-1}{u+1} .
$$

Since we are interested in $h_{0}(u)+h_{1}(u)$, we consider a linear differential equation for the sum,

$$
\frac{\partial\left(h_{0}+h_{1}\right)}{\partial u}-\frac{2 u}{u^{2}-1} \sum_{n=2} n \tilde{I}^{(n)}(S) \frac{\partial h_{1}}{\partial \tilde{I}^{(n)}(S)}=\gamma u
$$

where we used that $h_{0}$ does not depend on $\tilde{I}^{(n)}(S)$. Obviously $h_{0}+h_{1}$ can be expanded in powers of $\tilde{I}^{(n)}(S)$ and by comparing equal powers on the left- and on the right-hand side of the above equation we derive the $u$-dependence of the coefficient functions, which involve integration constants. An additional integration constant is determined by using that $h_{0}+h_{1}$ vanishes at $u=1$. The final answer for $h_{0}+h_{1}$ then takes the form,

$$
\begin{aligned}
h_{0}(u)+h_{1}(u)= & \frac{1}{2} \gamma\left(u^{2}-1\right)+\sum_{m=2} \alpha_{m}\left(u^{2}-1\right)^{m} \tilde{I}^{(m)}(S) \\
& +\sum_{m, n=2} \alpha_{m, n}\left(u^{2}-1\right)^{m+n} \tilde{I}^{(m)}(S) \tilde{I}^{(n)}(S) \\
& +\sum_{m, n, p=2} \alpha_{m, n, p}\left(u^{2}-1\right)^{m+n+p} \tilde{I}^{(m)}(S) \tilde{I}^{(n)}(S) \tilde{I}^{(p)}(S)+\cdots,
\end{aligned}
$$

where the coefficients $\alpha_{m, \ldots}$ are integration constants. Obviously there is a substantial simplification in the $u$-dependence of (B.6) as compared to the general terms that we found in section 6 . Although in the latter case all contributions vanish at the perturbative point $u=1$, the dependence on $u$ in section 6 is never solely expressed in powers of $u^{2}-1$. This behaviour is really the result of the large-moduli limit, which restricts all the $\tilde{I}^{(p)}(T)$ and $\tilde{I}^{(p)}(U)$ to constants. This induces an enormous rearrangement of terms that lead to (B.6). As we will see, we may compare the results by matching the coefficients of an expansion in powers of $u-1$.

To illustrate this, let us consider the expressions derived in section 6 and analyze how they contribute in the large-moduli limit to terms linear and quadratic in the $\tilde{I}^{(n)}(S)$. From (B.6) we know that the results can be expressed in an expansion in powers of $u-1$, 
where the lowest power is known. Likewise the coefficient functions that we determined in section 6 can also be expressed as a power series in $u-1$ and the lowest power should therefore coincide with the one indicated in (B.6). We have calculated the following terms in section 6 ,

$$
\begin{aligned}
& \sum_{m=2} c_{m}(u)\left[\tilde{I}^{(m)}(S)+\cdots\right] \\
+ & \sum_{m, n=2} d_{m, n}(u)\left[\tilde{I}^{(m)}(S) \tilde{I}^{(n)}(S)+\cdots\right] \\
+ & \sum_{m, n=2} e_{m, n}(u)\left[\tilde{I}^{(m)}(S) \tilde{I}^{(n)}(T)+\cdots\right] \\
+ & p(u)\left[\left(\tilde{I}^{(2)}(S)\right)^{2} \tilde{I}^{(2)}(T)+\cdots\right] \\
+ & v_{2,2,2}(u)\left[\tilde{I}^{(2)}(S) \tilde{I}^{(2)}(T) \tilde{I}^{(2)}(U)\right],
\end{aligned}
$$

where the ellipses denote the extra terms that follow from triality, while the $u$-dependence of the coefficient functions is known. Note that the above terms only represent a subset of all possible terms.

Let us first consider the terms linear in $\tilde{I}^{(n)}(S)$ that arise in the large-moduli limit of (B.7),

$$
\left[c_{n}(u)+\sum_{m=2} e_{n, m}(u) \frac{(m-1) !}{\gamma^{m-1}}\right] \tilde{I}^{(n)}(S)+\frac{1}{4 \gamma^{2}}\left[2 p(u)+v_{2,2,2}(u)\right] \tilde{I}^{(2)}(S) .
$$

By comparing powers of $u-1$ it follows that the lowest power of the above expression must be equal to $(u-1)^{n}$. However, the only term that is proportional to $(u-1)^{n}$ in the above expression is the first one equal to $c_{n}(u)$. Assuming that the above equation indeed contains all the relevant terms, it follows that $\alpha_{n}$ is directly related to the value of $(u-1)^{-n} c_{n}(u)$ at the perturbative point $u=1$. This yields the result,

$$
\alpha_{n}=\frac{\gamma^{n}}{2^{n} n !} .
$$

Based on (B.7) one can also analyze the terms of second order in $\tilde{I}^{(m)}(S)$ and compare them to $\alpha_{m, n}$. Here the large-moduli limit yields,

$$
\left[d_{2,2}(u)+\frac{1}{\gamma} p(u)\right]\left(\tilde{I}^{(2)}(S)\right)^{2}=\frac{(u-1)^{4}}{8}\left(\tilde{I}^{(2)}(S)\right)^{2}+\mathcal{O}\left((u-1)^{5}\right),
$$

where we made use of (6.12) and (6.18). Upon comparing this with the corresponding term in (B.6) we derive

$$
\alpha_{2,2}=\frac{\gamma^{3}}{128}
$$

provided that all relevant terms were included in (B.7). Using (B.7), one contribution to the values of the $\alpha_{m, n}$ for $m>2$ can be read off directly from the functions $d_{m, n}(u)$ given in (6.15). However, there may be further contributions to these values from terms that we 
have not computed, such as generalizations of the term proportional to $p(u)$ in (B.7) that involve $\tilde{I}^{(m)}(S)$ with $m>2$.

From the result (B.6) one can directly obtain the large-moduli limit of the function $\Omega$ that encodes the effective Wilsonian action by invoking (4.9). From (5.21) we have

$$
u^{2}-1=\frac{2 \lambda}{\gamma^{2}} D_{S} \omega D_{T} \omega D_{U} \omega \approx \frac{2 \lambda}{\gamma^{2}} \partial_{S} \omega \partial_{T} \omega \partial_{U} \omega
$$

where the second equation is the result of the large-moduli limit and we suppress nonholomorphic corrections. Upon including the terms linear in $\omega(S)+\omega(T)+\omega(U)$ we then obtain the following result,

$$
\begin{aligned}
h(\omega ; \lambda)= & \omega(S)+\omega(T)+\omega(U)+\frac{\lambda}{\gamma} \partial_{S} \omega \partial_{T} \omega \partial_{U} \omega \\
& +\sum_{m=2} \alpha_{m}\left(\frac{2 \lambda}{\gamma^{2}}\right)^{m}\left(\partial_{T} \omega \partial_{U} \omega\right)^{m} I^{(n)}(S) \\
& +\sum_{m, n=2} \alpha_{m, n}\left(\frac{2 \lambda}{\gamma^{2}}\right)^{m+n}\left(\partial_{T} \omega \partial_{U} \omega\right)^{m+n} I^{(m)}(S) I^{(n)}(S) \\
& +\sum_{m, n, p=2} \alpha_{m, n, p}\left(\frac{2 \lambda}{\gamma^{2}}\right)^{m+n+p}\left(\partial_{T} \omega \partial_{U} \omega\right)^{m+n+p} I^{(m)}(S) I^{(n)}(S) I^{(p)}(S)+\cdots
\end{aligned}
$$

which is holomorphic. According to (4.9), the above expression yields an expansion in terms of $\lambda$ with coefficients that can be compared to the functions $\omega^{(n)}(S, T, U)$ that appear in the function $\Omega$ as defined in (2.14). We can now use the known values for $\alpha_{2}, \alpha_{3}, \alpha_{4}$ and $\alpha_{2,2}$ to obtain the large-moduli limit of $\omega^{(n)}(S, T, U)$ with $n \leq 5$ and compare the result with the explicit results obtained in section 3 . The result takes the form

$$
\begin{aligned}
h(\omega ; \lambda)= & \omega(S)+\omega(T)+\omega(U)+\frac{\lambda}{\gamma} \partial_{S} \omega \partial_{T} \omega \partial_{U} \omega \\
& +\frac{\lambda^{2}}{2 \gamma^{2}}\left(\partial_{T} \omega \partial_{U} \omega\right)^{2} I^{(2)}(S)+\frac{\lambda^{3}}{6 \gamma^{3}}\left(\partial_{T} \omega \partial_{U} \omega\right)^{3} I^{(3)}(S) \\
& +\frac{\lambda^{4}}{24 \gamma^{4}}\left(\partial_{T} \omega \partial_{U} \omega\right)^{4}\left[I^{(4)}(S)+\frac{3}{\gamma}\left[I^{(2)}(S)\right]^{2}\right]+\mathcal{O}\left(\lambda^{5}\right)
\end{aligned}
$$

All these terms agree with the results that follow from the large-moduli limit applied to the corresponding functions $\omega^{(n)}(S, T, U)$ that were calculated in section 3 .

Open Access. This article is distributed under the terms of the Creative Commons Attribution License (CC-BY 4.0), which permits any use, distribution and reproduction in any medium, provided the original author(s) and source are credited. 


\section{References}

[1] A. Sen and C. Vafa, Dual pairs of type-II string compactification, Nucl. Phys. B 455 (1995) 165 [hep-th/9508064] [INSPIRE].

[2] A. Gregori, C. Kounnas and P.M. Petropoulos, Nonperturbative triality in heterotic and type-II N =2 strings, Nucl. Phys. B 553 (1999) 108 [hep-th/9901117] [INSPIRE].

[3] T.W. Grimm, A. Klemm, M. Mariño and M. Weiss, Direct Integration of the Topological String, JHEP 08 (2007) 058 [hep-th/0702187] [INSPIRE].

[4] B. de Wit and A. Van Proeyen, Potentials and Symmetries of General Gauged N=2 Supergravity: Yang-Mills Models, Nucl. Phys. B 245 (1984) 89 [InSPIRE].

[5] S. Cecotti, S. Ferrara and L. Girardello, Geometry of Type II Superstrings and the Moduli of Superconformal Field Theories, Int. J. Mod. Phys. A 4 (1989) 2475 [inSPIRE].

[6] G.L. Cardoso, B. de Wit and S. Mahapatra, Subleading and non-holomorphic corrections to $N=2$ BPS black hole entropy, JHEP 02 (2009) 006 [arXiv:0808.2627] [INSPIRE].

[7] G.L. Cardoso, B. de Wit and S. Mahapatra, BPS black holes, the Hesse potential and the topological string, JHEP 06 (2010) 052 [arXiv: 1003.1970] [INSPIRE].

[8] G.L. Cardoso, B. de Wit and S. Mahapatra, Non-holomorphic deformations of special geometry and their applications, Springer Proc. Phys. 144 (2013) 1 [arXiv:1206.0577] [INSPIRE].

[9] G.L. Cardoso, B. de Wit and S. Mahapatra, Deformations of special geometry: in search of the topological string, JHEP 09 (2014) 096 [arXiv: 1406.5478] [INSPIRE].

[10] M. Bershadsky, S. Cecotti, H. Ooguri and C. Vafa, Holomorphic anomalies in topological field theories, Nucl. Phys. B 405 (1993) 279 [hep-th/9302103] [INSPIRE].

[11] M. Bershadsky, S. Cecotti, H. Ooguri and C. Vafa, Kodaira-Spencer theory of gravity and exact results for quantum string amplitudes, Commun. Math. Phys. 165 (1994) 311 [hep-th/9309140] [INSPIRE].

[12] G.L. Cardoso and T. Mohaupt, Hessian geometry and the holomorphic anomaly, JHEP 02 (2016) 161 [arXiv:1511.06658] [INSPIRE].

[13] M. Alim, S.-T. Yau and J. Zhou, Airy Equation for the Topological String Partition Function in a Scaling Limit, Lett. Math. Phys. 106 (2016) 719 [arXiv:1506.01375] [INSPIRE].

[14] B. de Wit, S. Katmadas and M. van Zalk, New supersymmetric higher-derivative couplings: Full $N=2$ superspace does not count!, JHEP 01 (2011) 007 [arXiv:1010.2150] [INSPIRE].

[15] D. Butter, B. de Wit, S.M. Kuzenko and I. Lodato, New higher-derivative invariants in $N=2$ supergravity and the Gauss-Bonnet term, JHEP 12 (2013) 062 [arXiv:1307.6546] [INSPIRE].

[16] A. Dabholkar, F. Denef, G.W. Moore and B. Pioline, Precision counting of small black holes, JHEP 10 (2005) 096 [hep-th/0507014] [INSPIRE].

[17] R. Gopakumar and C. Vafa, M theory and topological strings. 1, hep-th/9809187 [INSPIRE].

[18] R. Gopakumar and C. Vafa, M theory and topological strings. 2, hep-th/9812127 [INSPIRE].

[19] G.L. Cardoso, B. de Wit, J. Käppeli and T. Mohaupt, Stationary BPS solutions in $N=2$ supergravity with $R^{2}$ interactions, JHEP 12 (2000) 019 [hep-th/0009234] [INSPIRE]. 
[20] F. Denef and G.W. Moore, Split states, entropy enigmas, holes and halos, JHEP 11 (2011) 129 [hep-th/0702146] [INSPIRE].

[21] A. Sen, Logarithmic Corrections to $N=2$ Black Hole Entropy: An Infrared Window into the Microstates, Gen. Rel. Grav. 44 (2012) 1207 [arXiv:1108.3842] [INSPIRE].

[22] M. Dedushenko and E. Witten, Some Details On The Gopakumar-Vafa and Ooguri-Vafa Formulas, Adv. Theor. Math. Phys. 20 (2016) 1 [arXiv:1411.7108] [InSPIRE].

[23] H. Hahn, Eisenstein series associated with $\Gamma_{0}(2)$, Ramanujan J. 15 (2008) 235 [arXiv: 1507.04425].

[24] G.L. Cardoso, S. Nampuri and D. Polini, An approach to BPS black hole microstate counting in an $N=2$ STU model, arXiv: 1903.07586 [INSPIRE].

[25] G.L. Cardoso, B. de Wit, J. Käppeli and T. Mohaupt, Black hole partition functions and duality, JHEP 03 (2006) 074 [hep-th/0601108] [INSPIRE]. 